\title{
Adiabatic quantum games and phase-transition-like behavior between optimal strategies
}

\author{
M. A. de Ponte $^{1}\left(\right.$ D $\cdot$ Alan C. Santos ${ }^{2}$
}

Received: 26 February 2018 / Accepted: 27 April 2018 / Published online: 10 May 2018

C Springer Science+Business Media, LLC, part of Springer Nature 2018

\begin{abstract}
In this paper we propose a game of a single qubit whose strategies can be implemented adiabatically. In addition, we show how to implement the strategies of a quantum game through controlled adiabatic evolutions, where we analyze the payment of a quantum player for various situations of interest: (1) when the players receive distinct payments, (2) when the initial state is an arbitrary superposition, and (3) when the device that implements the strategy is inefficient. Through a graphical analysis, it is possible to notice that the curves that represent the gains of the players present a behavior similar to the curves that give rise to a phase transition in thermodynamics. These transitions are associated with optimal strategy changes and occur in the absence of entanglement and interaction between the players.
\end{abstract}

Keywords Quantum games · Adiabatic evolution · Phase-transition-like behavior

\section{Introduction}

The games theory studies mathematical models in which two or more players aim to maximize their gains by implementing different strategies. This theory can be applied in several areas of knowledge, including studies of animal behavior [1], evolution

M. A. de Ponte

maponte@itapeva.unesp.br

Alan C. Santos

ac_santos@id.uff.br

1 Universidade Estadual Paulista (UNESP), Campus Experimental de Itapeva, Itapeva, São Paulo 18409-010, Brazil

2 Instituto de Física, Universidade Federal Fluminense, Av. Gal. Milton Tavares de Souza s/n, Gragoatá, Niterói, Rio de Janeiro 24210-346, Brazil 
of species by natural selection [2,3], social sciences [4-6], political sciences [7-10], military sciences [11], ethics [12,13], economy [14-17], philosophy [18,19], and, more recently, has awakening the attention of computer science that has been using it in advances in artificial intelligence and cybernetics [20-23]. The quantum version of games theory has recently become an active branch of research which gain new adepts every year. In addition, the quantum game theory can be useful for finding more efficient algorithms for quantum computers [24] and quantum communication protocols [25]. As occurred in the classical theory, the quantum version has been extended to other areas, as for example, in biology where the researches on protein molecules are turning to a completely quantum description [26].

On the other hand, the use of adiabatic evolutions has recently awaked interest as a promising model in quantum computation that implements quantum algorithms and performs quantum simulations in several systems: qubit superconductors [27-30], trapped ions [31,32], nuclear spin on nuclear magnetic resonance [33-35], among others $[36,37]$. Such implementations are based on the adiabatic theorem of quantum mechanics [38-40], and the regimes of validity [41-45] were experimentally discussed in reference [46].

In this work, we joint these two themes (games theory and adiabatic evolutions) in a game of a single qubit ("even or odd", "heads or tails", "spin flip", etc.). To accomplish this task, we show how to implement the strategies through to the formalism of adiabatic evolutions and, as a consequence, we introduce a parameter that allows us to simulate the efficiency of a machine when implementing each of the game strategies. Next, we provide a general analysis to get the optimal strategies for various regimes of system parameter, including the parameters that define the initial state of the game and the payments of each player. Finally, we show the existence of regions where the transition from one optimal strategy to another is similar to the phase transitions in thermodynamics, showing that these transitions are due to the state superposition of a single qubit and not necessarily due to entanglement between the qubits of the output game state, as studied in the reference [47].

\section{Adiabatic strategies}

The purpose of this section is to introduce the reader to the basic concepts that we should use in this work such that reading and understanding become as smooth as possible. We will start this section by introducing the concepts of strategies (classical and quantum) and adiabatic evolutions. In the end, we show how to implement strategies through controlled adiabatic evolutions, introduced by Hen [48].

\subsection{Classical and quantum strategies}

In games theory, each strategy is associated with an action that a group or even a single player can employ. In the quantum version of this theory, a strategy can represent the interaction between the quantum and auxiliary systems, which can be discarded at the end of the game. During the creation of a game, we construct rules in which we define the allowed operations (state evolutions of the game according to a set of 
Hamiltonians, measurements on an auxiliary system, etc.), the order of each participant in the game, and the payments (or gains) of each player related to one or more states of an orthonormal basis, which we should call the basis of game states $\left\{\left|\psi_{i}\right\rangle\right\}$, such that $\left\langle\psi_{j} \mid \psi_{i}\right\rangle=\delta_{i j}$. In a genuinely classical game, the state associated with the game must at any time be a statistical mixture of base states, and therefore, the density operator of the system must be diagonal, i.e., $\rho=\sum_{i} p_{i}\left|\psi_{i}\right\rangle\left\langle\psi_{i}\right|$, where $p_{i}$ is the probability of measuring the state $\left|\psi_{i}\right\rangle$, such that $\operatorname{Tr} \rho=\sum_{i} p_{i}=1$. Note that a classical strategy cannot give rise to non-diagonal elements of this density operator, whereas a quantum strategy does. For this reason, we must conclude that a classical strategy should allow only that the game's input state, let's say a element $\left|\psi_{j}\right\rangle\left\langle\psi_{j}\right|$, "jump" or "rotate" to an output state $\left|\psi_{k}\right\rangle\left\langle\psi_{k}\right|$, where $k \neq j$. To make clear what we have just said, consider the basis of the game as the computational basis of a single qubit $\{|0\rangle,|1\rangle\}$ in which one of the players gains $x_{0} \$$, if the final state is $|0\rangle$, and the other $x_{1} \$$, if the final state is $|1\rangle$. A strategy will be considered classical if the state of the game remains unchanged or rotate, $|0\rangle$ to $|1\rangle$ or $|1\rangle$ to $|0\rangle$, without introduction of relative phases. These relative phases can not exist because a classical player cannot access the non-diagonal elements of the density operator, restricted only to quantum players. On the other hand, a quantum strategy must take any state of the base $\{|0\rangle,|1\rangle\}$ into a superposition or introduce phases in the base states, i.e., $|0\rangle \rightarrow e^{i \delta_{0}}|0\rangle$ or $|1\rangle \rightarrow e^{i \delta_{1}}|1\rangle$. If in the latter case $\delta_{0}-\delta_{1} \neq 0,2 \pi, \ldots$ and the input state of the game is a superposition, then the relative phase between them is modified and consequently will be exist a change in the non-diagonal elements of the density operator.

\subsection{Adiabatic evolutions}

Since each player does not know the game state before implementing his strategy, any protocol that implements it should be independent of that state. Therefore, to implement a quantum game in the adiabatic form, we should be able to perform an operation in any state of a qubit. This adiabatic process requires degenerate Hamiltonians, as we shall see below.

The adiabatic evolution is generally defined by means of a Hamiltonian $\mathcal{H}(s)$ which varies slowly over time and interpolates two time-independent Hamiltonians, $\mathcal{H}_{\text {in }}$ and $\mathcal{H}_{\text {out }}$, such that $\left[\mathcal{H}_{\text {in }}, \mathcal{H}_{\text {out }}\right] \neq 0$, being mathematically written in the form

$$
\mathcal{H}(s)=f(s) \mathcal{H}_{\text {in }}+g(s) \mathcal{H}_{\text {out }},
$$

where $s=t / \tau$ is a dimensionless time variable and $\tau$ represents the complete time evolution. The real functions $f$ and $g$ in (2) must satisfy the boundary conditions $f(0)=g(1)=1$ and $f(1)=g(0)=0$. According to the controlled adiabatic dynamics, the Hamiltonian $\mathcal{H}(s)$, at $s=0$, must acts on an eigenstate of $\mathcal{H}_{\text {in }} \equiv$ $\mathbb{1}_{\text {tar }} \otimes \mathcal{H}_{\text {aux }}$ and drive it, at $s=1$, for an eigenstate of $\mathcal{H}_{\text {out }}$. If the auxiliary system is initially in the eigenstate $\left|0_{\text {aux }}\right\rangle$, note that the initial state of the composite system $\left|\Psi_{\text {in }}\right\rangle=\left|\psi_{\operatorname{tar}}(0)\right\rangle \otimes\left|0_{\text {aux }}\right\rangle$ is a eigenstate of $\mathcal{H}_{\text {in }}$ for whichever the state $\left|\psi_{\operatorname{tar}}(0)\right\rangle$ is. On the other hand, if $\mathcal{H}_{\text {out }}$ represents the interaction between the auxiliary and target 
systems, the output state of the composite system should be, in general, entangled. However, if $\mathcal{H}_{\text {out }}=\mathcal{H}_{\text {tar }} \otimes \mathcal{H}_{\text {aux }}^{\prime}$, the output state after the adiabatic evolution will be disentangled and of the form $\left|\Psi_{\text {out }}\right\rangle=\left|\psi_{\text {tar }}(1)\right\rangle \otimes\left|0_{\text {aux }}^{\prime}\right\rangle$, where $\left|0_{\text {aux }}^{\prime}\right\rangle$ is an eigenstate of $\mathcal{H}_{\text {aux }}^{\prime}$, which is not necessarily equal to $\mathcal{H}_{\text {aux }}$, and $\left|\psi_{\text {tar }}(1)\right\rangle$ is an eigenstate of $\mathcal{H}_{\text {tar }}$, which is defined according to the operation to be performed on $\left|\psi_{\operatorname{tar}}(0)\right\rangle$.

In the specific case of a game, we define an adiabatic strategy as being an operation on the game state performed through the adiabatic evolution that follows from the Hamiltonian defined in (1).

\subsection{Using controlled adiabatic evolutions in the implementation of strategies}

Based on the fact that quantum games, which are being developed and studied in the literature, can be implemented efficiently through circuit models, we will redirect the study of adiabatic strategies to the situation in which adiabatic evolution is performed in a controlled way, as proposed by Hen [48]. In this work, Hen demonstrates that a single qubit logic gate (which is nothing more than a unitary operation that can be seen as a rotation by an angle $\phi$ in the vicinity of an arbitrary direction $\hat{n}$ in the Bloch sphere) can be adiabatically implemented using controlled evolutions by means of the two-qubit temporal Hamiltonian, defined by

$$
\mathcal{H}(s)=\mathbb{P}_{+} \otimes \mathcal{H}_{0}(s)+\mathbb{P}_{-} \otimes \mathcal{H}_{\phi}(s)
$$

In Eq. (2), the operators $\mathbb{P}_{ \pm}$represent a complete set of projectors mutually orthogonal that act on the Hilbert space of the target qubit (system of interest) and can be written in terms of an arbitrary direction $\hat{n}$ as $\mathbb{P}_{ \pm}=(\mathbb{1} \pm \hat{n} \cdot \vec{\sigma}) / 2$, where $\vec{\sigma}=\sigma_{x} \hat{x}+\sigma_{y} \hat{y}+\sigma_{z} \hat{z}$ and whose matrices $\sigma_{x}, \sigma_{y}, \sigma_{z}$ are the Pauli matrices. The Hamiltonian $\mathcal{H}_{\phi}(s)$, which appears in (2), is defined by

$$
\mathcal{H}_{\phi}(s)=-\hbar \omega\left\{\cos \left(\theta_{0} s\right) \sigma_{z}+\sin \left(\theta_{0} s\right)\left[\sigma_{x} \cos \phi+\sigma_{y} \sin \phi\right]\right\}
$$

such that $\mathcal{H}_{0}(s)=\mathcal{H}_{\phi=0}(s)$.

In this way, if the initial state of the composite system, represented by $|\Psi(0)\rangle=$ $\left|\psi_{\text {tar }}\right\rangle \otimes\left|0_{\text {aux }}\right\rangle \equiv|\psi ; 0\rangle$, evolves adiabatically through (2), the final state $|\Psi(s)\rangle$, according to [48], becomes

$$
|\Psi(s)\rangle=\cos \left(\theta_{0} s / 2\right)|\psi ; 0\rangle+\sin \left(\theta_{0} s / 2\right)\left|\psi_{\text {rot }} ; 1\right\rangle
$$

where $\left|\psi_{\text {rot }}\right\rangle=\mathbf{U}(\phi ; \hat{n})|\psi\rangle$ represents a rotation about the initial state. As observed by Hen [48], $\mathbf{U}(\phi ; \hat{n})$ can implement any unitary rotation under the initial state of the target qubit $|\psi\rangle$ and therefore can implement any strategy, whether classical or not. In this way, there always will be an adiabatic Hamiltonian who is able to implement such strategies.

Note that in general the final state $|\Psi(s)\rangle$ in (4) is an entangled state of the composite system and therefore the target qubit state will depend on the result of the measure under the state of auxiliary qubit. If the result of this measure is $\left|0_{\text {aux }}\right\rangle$, then the target 
qubit state will be the same as the initial state $|\psi\rangle$ (in this case, the implementation of the operation $\mathbf{U}(\phi, \hat{n})$ on the target qubit state was not successful). However, if the result of the measurement is $\left|1_{\text {aux }}\right\rangle$, then the operation $\mathbf{U}(\phi, \hat{n})$ was implemented on the target qubit state successfully. Therefore, it is easy to conclude that the meaning of $\theta_{0}$ is related to the probability of obtaining the state $\left|\psi_{\text {rot }}\right\rangle$. In fact, we can see from (4) that $|\psi\rangle$ and $\left|\psi_{\text {rot }}\right\rangle$ are obtained with a probability of $\cos ^{2}\left(\theta_{0} / 2\right)$ and $\sin ^{2}\left(\theta_{0} / 2\right)$, respectively.

Since in general the adiabatic strategy is implemented probabilistically (being deterministic only when $\theta_{0}=\pi$ ), we can simulate some imperfection in the machine, which performs the game strategic operations, through the parameter $\theta_{0}$. In fact, when we measure the state of the auxiliary system, the game state becomes a statistical mixture of the form

$$
\rho=\cos ^{2}\left(\theta_{0} / 2\right)|\psi ; 0\rangle\left\langle\psi ; 0\left|+\sin ^{2}\left(\theta_{0} / 2\right)\right| \psi_{\text {rot }} ; 1\right\rangle\left\langle\psi_{\text {rot }} ; 1\right|,
$$

which is the same as in a classical situation where the machine or device that implements the strategy works in about $\sin ^{2}\left(\theta_{0} / 2\right)$ of the times. As a consequence of this interpretation of $\theta_{0}$, it is natural to conclude that the analysis of the best adiabatic strategies will differ from those in which the game is performed deterministically, as we will see in the next section.

\section{Quantum games with controlled adiabatic evolutions}

Before we start to analyze the optimal strategies in an adiabatic quantum game, we let's review some important points of a "heads or tails" quantum game.

\subsection{Two-person quantum game: "heads or tails"}

In a two-person quantum game with zero sum (that is, what one player gains is what the other loses), the goal is to guess the final state of the coin ("heads", which we represent by state $|0\rangle$, or "tails", represented by the state $|1\rangle$ ). During the course of the game, both players (the quantum player Alice and the classic player Bob) can use strategies to change the coin state.

The advantage that a quantum player has over a classic, mentioned in Meyer's original work [49], can be easily understood in the situation where the coin initial state is a superposition of the form $\left|\psi_{c}\right\rangle=(|0\rangle+|1\rangle) / \sqrt{2}$. If the game starts with Bob and he is restricted to the use of classical strategies $\left\{\mathbb{1}, \sigma_{x}\right\}$, we see that the initial game state does not change, since $\sigma_{x}$ has the effect of rotating the state from $|0\rangle$ to $|1\rangle$ and vice versa. Therefore, if Alice uses the strategy $\mathcal{H}=\left(\sigma_{x}+\sigma_{z}\right) / \sqrt{2}=\mathrm{H}$, known as the Hadamard operator, the final state becomes $|0\rangle$. On the other hand, if $\mathcal{H}=\mathrm{H} \sigma_{z}$ the final state will be $|1\rangle$. Since Alice is able to control the final game state, we conclude that the use of quantum strategies allows her to always win Bob and receive the maximum prize of the game. In situations where both players are classic, any player will have a $50 \%$ chance of winning and therefore the net amount at the end of numerous disputes will be null, in which case we say that the game is fair. 
In the other situation, when the initial game state is $|0\rangle$ or $|1\rangle$, we note that after Bob's movement, the state will be $|0\rangle$ or $|1\rangle$ and therefore it is impossible for there to be a unitary operator that takes both states to the same final state, which prevents Alice's victory at all times. This impossibility can disappear if we allow the use of strategies associated with non-unitary and/or non-Hermitian operations. This study is far from the scope of this paper and could be approached in future research with the use of adiabatic evolutions for non-Hermitian Hamiltonians [50,51] and/or adiabatic evolutions in open systems [41].

\subsubsection{Analyzing the advantage of the quantum player in the adiabatic case}

In the adiabatic version of the "heads or tails" game, which we will see in detail in the next section, we will consider the same rules we previously assumed. In addition, the scheme for the adiabatic game will consist of the target qubit, which will represent the coin state (or game state), and two auxiliary qubits: one in exclusive possession of Bob and the other in exclusive possession of Alice. When necessary, we will assign the indices " $c$ ", " $B$ " and " $A$ " to denote the qubits of the coin, Bob and Alice respectively. Since the final state of the adiabatic evolution, in general, is an entangled state of the target qubit + auxiliary qubit, when we measured the auxiliary qubit, the game state becomes a statistical mixture, except for a few particular cases which we describe below.

If the initial game state is $\left|\psi_{c}\right\rangle=\left(\left|0_{c}\right\rangle+\left|1_{c}\right\rangle\right) / \sqrt{2}$ and Bob, who is the first to play, performs a classic adiabatic move $\mathbb{1}_{B}$ or $\sigma_{x ; B}$, then by setting $\theta_{0}=\theta_{B}$ in (4), we see that the state of Bob's auxiliary qubit + coin state becomes

$$
\left|\Psi_{c B}\right\rangle=\left|\psi_{c}\right\rangle \otimes\left[\cos \left(\theta_{B} / 2\right)\left|0_{B}\right\rangle+\sin \left(\theta_{B} / 2\right)\left|1_{B}\right\rangle\right]
$$

where we use the fact that $\left|\psi_{\text {rot }}\right\rangle=\left|\psi_{c}\right\rangle$ for Bob's classical moves.

Note from (6) that the auxiliary state is disentangled from the coin state, and therefore any result of the measurement on the auxiliary qubit leaves the state of the coin in $\left|\psi_{c}\right\rangle$, and, consequently, we can exclude the state of Bob's auxiliary qubit. If now Alice, which is the second and the last to play, implements a Hadamard $\mathrm{H}$ operation, we will see that the state of Alice's auxiliary qubit + coin state, redefining $\theta_{0}=\theta_{A}$ in (4), will be given by

$$
\left|\Psi_{c A}\right\rangle=\cos \left(\theta_{A} / 2\right)\left|\psi_{c}\right\rangle \otimes\left|0_{A}\right\rangle+\sin \left(\theta_{A} / 2\right)\left|0_{c}\right\rangle \otimes\left|1_{A}\right\rangle
$$

where we use the fact that $\left|\psi_{\text {rot }}\right\rangle=\mathrm{H}\left|\psi_{c}\right\rangle=\left|0_{c}\right\rangle$. Observe that when we measure the state of Alice's auxiliary qubit we have a probability of $\cos ^{2}\left(\theta_{A} / 2\right)$ to measure $\left|0_{A}\right\rangle$ and a probability of $\sin ^{2}\left(\theta_{A} / 2\right)$ to measure $\left|1_{A}\right\rangle$.

When the game is played multiple times, we can write the coin state as a statistical mixture $\rho_{c}$ of the results obtained above, so that

$$
\begin{aligned}
\rho_{c} & =\operatorname{Tr}_{A}\left|\Psi_{c A}\right\rangle\left\langle\Psi_{c A}\right| \\
& =\cos ^{2}\left(\theta_{A} / 2\right)\left|\psi_{c}\right\rangle\left\langle\psi_{c}\left|+\sin ^{2}\left(\theta_{A} / 2\right)\right| 0_{c}\right\rangle\left\langle 0_{c}\right|
\end{aligned}
$$


From (8), we see that the probability of finding the target qubit in the state $\left|0_{c}\right\rangle$ becomes $p_{0}=1-\cos ^{2}\left(\theta_{A} / 2\right) / 2$, whereas the probability of finding the state $\left|1_{c}\right\rangle$ will be $p_{1}=\cos ^{2}\left(\theta_{A} / 2\right) / 2$. Thus, if Alice gains (loses) an amount $\$$ if the final coin state is $\left|0_{c}\right\rangle\left(\left|1_{c}\right\rangle\right)$, her net gain or payment $\pi_{A}$ will be, after countless moves, around of

$$
\pi_{A}=p_{0} \$-p_{1} \$=\sin ^{2}\left(\theta_{A} / 2\right) \$,
$$

which is always greater than zero, since in adiabatic evolutions $\theta_{A} \neq 0$ (otherwise, no evolution will ever be implemented). The symbol \$ represents the payment value for Alice in cases where the coin state is $\left|0_{c}\right\rangle$ (we assume that Alice loses the same amount when the final state is $\left|1_{c}\right\rangle$ ). Note, therefore, that the payment of Alice becomes maximum and equal to the previous game only when $\theta_{A}=\pi$, in which case the controlled adiabatic evolution ceases to be probabilistic and the implementation of unitary operation is achieved with $100 \%$ of success.

\subsection{Adiabatic version of the two-person quantum game: "heads or tails"}

In the previous section we investigate how the advantage of a player, using quantum strategies, changes in the adiabatic version of Meyer's original game. In this simplified analysis, we focused only on quantum strategy, ignoring Alice's classical strategies in our study. In this section, therefore, in addition to taking into account the classic and quantum strategies for both players, we are interested in analyzing the game for general situations in which the players' gains are different and when the initial game state is a superposition rather than a single state of the game base.

To carry out these analyzes, let us consider the general situation in which the initial coin state is a superposition of the form

$$
\left|\varphi_{c}\right\rangle=\alpha_{0}\left|0_{c}\right\rangle+\alpha_{1}\left|1_{c}\right\rangle
$$

with the normalization condition $\left|\alpha_{0}\right|^{2}+\left|\alpha_{1}\right|^{2}=1$.

Both players can use strategies $\left\{\mathbb{1}, \sigma_{x}, \mathbf{U}\right\}$, where the first two are classic strategies and the latter is an arbitrary strategy that performs unitary rotations such that:

$$
\begin{aligned}
& \mathbf{U}_{\ell}\left|0_{c}\right\rangle=\cos \left(\phi_{\ell} / 2\right)\left|0_{c}\right\rangle+e^{i \delta_{\ell}} \sin \left(\phi_{\ell} / 2\right)\left|1_{c}\right\rangle \\
& \mathbf{U}_{\ell}\left|1_{c}\right\rangle=\sin \left(\phi_{\ell} / 2\right)\left|0_{c}\right\rangle-e^{i \delta_{\ell}} \cos \left(\phi_{\ell} / 2\right)\left|1_{c}\right\rangle,
\end{aligned}
$$

where the index $\ell=A, B$ labels the strategies of Alice and Bob. Note that for cases where $\phi_{\ell}=0$, with $\delta_{\ell}=\pi$, and $\phi_{\ell}=\pi$, with $\delta_{\ell}=0$, we retrieve, respectively, the classical strategies $\mathbb{1}$ and $\sigma_{x}$ from $\mathbf{U}$. For any other situation, $\mathbf{U}$ will represent a quantum strategy. Since $\mathbf{U}$, defined in (11), is very general, we will now analyze the adiabatic evolution of the initial state (10) through $\mathbf{U}$ only, and later we will particularize for some situations of interest.

Recalling that the system is composed of a coin qubit and the qubits of the auxiliary systems let's we will consider that the initial state of this system be $\left|\Psi_{C A B}(0)\right\rangle=$ $\left|\varphi_{c}\right\rangle \otimes\left|0_{B}, 0_{A}\right\rangle$. Assuming that Bob, being the first to play, uses the strategy $\mathbf{U}_{B}$, we see from (4), that the state of the system, after Bob's move, will be 


$$
\left|\Psi_{C A B}\right\rangle=\cos \left(\theta_{B} / 2\right)\left|\varphi_{c}\right\rangle \otimes\left|0_{B}, 0_{A}\right\rangle+\sin \left(\theta_{B} / 2\right) \mathbf{U}_{B}\left|\varphi_{c}\right\rangle \otimes\left|1_{B}, 0_{A}\right\rangle
$$

Then Alice implements the strategy $\mathbf{U}_{A}$ and, consequently, the final state of the composite system becomes:

$$
\begin{aligned}
\left|\Psi_{C A B}\right\rangle= & \cos \left(\theta_{A} / 2\right)\left[\cos \left(\theta_{B} / 2\right)\left|\varphi_{c}\right\rangle \otimes\left|0_{B}, 0_{A}\right\rangle\right. \\
& \left.+\sin \left(\theta_{B} / 2\right) \mathbf{U}_{B}\left|\varphi_{c}\right\rangle \otimes\left|1_{B}, 0_{A}\right\rangle\right] \\
& +\sin \left(\theta_{A} / 2\right)\left[\cos \left(\theta_{B} / 2\right) \mathbf{U}_{A}\left|\varphi_{c}\right\rangle \otimes\left|0_{B}, 1_{A}\right\rangle\right. \\
& \left.+\sin \left(\theta_{B} / 2\right) \mathbf{U}_{A} \mathbf{U}_{B}\left|\varphi_{c}\right\rangle \otimes\left|1_{B}, 1_{A}\right\rangle\right] .
\end{aligned}
$$

Finally, by performing the measurements of the auxiliary qubits of both players, we find that the coin state is taken to a statistical mixture of the form

$$
\begin{aligned}
\rho_{c}\left(\mathbf{U}_{A}, \mathbf{U}_{B}\right) \equiv & \rho_{c}(\mathbf{U}, \mathbf{U})=\operatorname{Tr}_{A, B}\left|\Psi_{c A B}\right\rangle\left\langle\Psi_{c A B}\right| \\
= & \cos ^{2}\left(\theta_{A} / 2\right)\left[\cos ^{2}\left(\theta_{B} / 2\right)\left|\varphi_{c}\right\rangle\left\langle\varphi_{c}\right|\right. \\
& \left.+\sin ^{2}\left(\theta_{B} / 2\right) \mathbf{U}_{B}\left|\varphi_{c}\right\rangle\left\langle\varphi_{c}\right| \mathbf{U}_{B}^{\dagger}\right] \\
& +\sin ^{2}\left(\theta_{A} / 2\right)\left[\cos ^{2}\left(\theta_{B} / 2\right) \mathbf{U}_{A}\left|\varphi_{c}\right\rangle\left\langle\varphi_{c}\right| \mathbf{U}_{A}^{\dagger}\right. \\
& \left.+\sin ^{2}\left(\theta_{B} / 2\right) \mathbf{U}_{A} \mathbf{U}_{B}\left|\varphi_{c}\right\rangle\left\langle\varphi_{c}\right| \mathbf{U}_{B}^{\dagger} \mathbf{U}_{A}^{\dagger}\right] .
\end{aligned}
$$

From the final coin state (13) and assuming that Alice receives from Bob a payment of $x_{0} \$$, when the coin state is measured in $\left|0_{c}\right\rangle$, and pays to Bob the amount $x_{1} \$$, when the coin state is $\left|1_{c}\right\rangle$, we can conclude that the average gain of Alice (in units of $\$$ ) will become

$$
\begin{aligned}
\pi_{A}(\mathbf{U}, \mathbf{U})= & \sum_{k=0}^{1}(-1)^{k} x_{k}\left\{\operatorname { c o s } ^ { 2 } ( \frac { \theta _ { A } } { 2 } ) \left[\cos ^{2}\left(\frac{\theta_{B}}{2}\right)\left|\alpha_{k}\right|^{2}\right.\right. \\
& \left.+\sin ^{2}\left(\frac{\theta_{B}}{2}\right)\left|\left\langle k\left|\mathbf{U}_{B}\right| \varphi_{c}\right\rangle\right|^{2}\right] \\
& +\sin ^{2}\left(\frac{\theta_{A}}{2}\right)\left[\cos ^{2}\left(\frac{\theta_{B}}{2}\right)\left|\left\langle k\left|\mathbf{U}_{A}\right| \varphi_{c}\right\rangle\right|^{2}\right. \\
& \left.\left.+\sin ^{2}\left(\frac{\theta_{B}}{2}\right)\left|\left\langle k\left|\mathbf{U}_{A} \mathbf{U}_{B}\right| \varphi_{c}\right\rangle\right|^{2}\right]\right\}
\end{aligned}
$$

By calculating the expected values that appear in (14) with the help of (11), we can rewrite $\pi_{A}(\mathbf{U}, \mathbf{U})$ as

$$
\begin{aligned}
\pi_{A}(\mathbf{U}, \mathbf{U})= & \frac{x_{0}-x_{1}}{2}+\left(x_{0}+x_{1}\right)\left\{\frac{\left|\alpha_{0}\right|^{2}-\left|\alpha_{1}\right|^{2}}{2} \Phi_{A} \Phi_{B}\right. \\
& +\operatorname{Re}\left(\alpha_{0} \alpha_{1}^{*}\right)\left[\sin \phi_{A} \sin ^{2}\left(\frac{\theta_{A}}{2}\right)\right.
\end{aligned}
$$




$$
\begin{aligned}
& \left.+\Phi_{A} \sin \phi_{B} \sin ^{2}\left(\frac{\theta_{B}}{2}\right)\right]+\sin \phi_{A} \sin ^{2}\left(\frac{\theta_{A}}{2}\right) \\
& \times \sin ^{2}\left(\frac{\theta_{B}}{2}\right)\left[\frac{\left|\alpha_{0}\right|^{2}-\left|\alpha_{1}\right|^{2}}{2} \sin \phi_{B} \cos \delta_{B}\right. \\
& -\operatorname{Re}\left(\alpha_{0} \alpha_{1}^{*}\right)\left(1+\cos \phi_{B} \cos \delta_{B}\right) \\
& \left.\left.-\operatorname{Im}\left(\alpha_{0} \alpha_{1}^{*}\right) \sin \delta_{B}\right]\right\},
\end{aligned}
$$

where we define the parameters

$$
\Phi_{\ell}=1-2 \sin ^{2}\left(\theta_{\ell} / 2\right) \sin ^{2}\left(\phi_{\ell} / 2\right) .
$$

\subsubsection{The role of the parameters $\theta_{A}$ and $\theta_{B}$ in a adiabatic classic game}

In the situations where it is desired that the quantum game is an extension of the classic game, it is necessary to fit or delimit some initially free parameters in a quantum game. Therefore, the purpose of this section is to only impose constraints on the parameters $\theta_{A}$ and $\theta_{B}$ through the analysis of a purely classical game.

Since in a purely classical game the initial state cannot be a superposition of base states, then we see that $\alpha_{0}$ or $\alpha_{1}$ must be null. In addition, both players should use only classical strategies (i.e., $\phi_{\ell}=0$, with $\delta_{\ell}=\pi$, and $\phi_{\ell}=\pi$, with $\delta_{\ell}=0$ ). Under these considerations, defining the parameters

$$
\begin{aligned}
& \pi_{+}(\mathbb{1}, \mathbb{1})=x_{0} \$ \\
& \pi_{-}(\mathbb{1}, \mathbb{1})=-x_{1} \$ \\
& \pi_{ \pm}(\mathbb{1}, \mathbf{x})=\left(\frac{x_{0}-x_{1}}{2} \pm \frac{x_{0}+x_{1}}{2} \cos \theta_{B}\right) \$ \\
& \pi_{ \pm}(\mathbf{x}, \mathbb{1})=\left(\frac{x_{0}-x_{1}}{2} \pm \frac{x_{0}+x_{1}}{2} \cos \theta_{A}\right) \$ \\
& \pi_{ \pm}(\mathbf{x}, \mathbf{x})=\left(\frac{x_{0}-x_{1}}{2} \pm \frac{x_{0}+x_{1}}{2} \cos \theta_{A} \cos \theta_{B}\right) \$,
\end{aligned}
$$

one can create a pay table with (15) for all possible combinations of players' strategies and that we show below for the initial states $|0\rangle$, Table 1 , and $|1\rangle$, Table 2 :

Observe that Table 2 can be obtained from Table 1 by making the mutual exchange $x_{0} \rightarrow-x_{1}$ and $x_{1} \rightarrow-x_{0}$, so that the game analysis for Table 1 is valid for Table 2 .

Table 1 Payments for Alice, according to the combination of players' strategies, in the situation where the initial game state is $|0\rangle$

\begin{tabular}{llll}
\hline$|\psi(0)\rangle=|0\rangle$ & Bob & & \\
\hline Alice & Strategies & $\mathbb{1}$ & $\sigma_{x}$ \\
& $\mathbb{1}$ & $\pi_{+}(\mathbb{1}, \mathbb{1})$ & $\pi_{+}(\mathbb{1}, \mathbf{x})$ \\
& $\sigma_{x}$ & $\pi_{+}(\mathbf{x}, \mathbb{1})$ & $\pi_{+}(\mathbf{x}, \mathbf{x})$ \\
\hline
\end{tabular}


Table 2 Payments for Alice, according to the combination of players' strategies, in the situation where the initial game state is $|1\rangle$

\begin{tabular}{llll}
\hline$|\psi(0)\rangle=|1\rangle$ & Bob & & \\
\hline Alice & Strategies & $\mathbb{1}$ & $\sigma_{x}$ \\
& $\mathbb{1}$ & $\pi_{-}(\mathbb{1}, \mathbb{1})$ & $\pi_{-}(\mathbb{1}, \mathbf{x})$ \\
& $\sigma_{x}$ & $\pi_{-}(\mathbf{x}, \mathbb{1})$ & $\pi_{-}(\mathbf{x}, \mathbf{x})$
\end{tabular}

In possession of a pay table, now we can do the analysis of the game, but before, we will define the concept of dominant strategies.

We say that $A_{j}$ weakly dominates the strategy $A_{k}\left(A_{j}\right.$ and $A_{k}$ are two arbitrary strategies of a player $A$ ) whenever the payment $\pi\left(A_{j}, B_{\ell}\right) \geq \pi\left(A_{k}, B_{\ell}\right)$, for any strategy $B_{\ell}$ that his opponent uses. If $\pi\left(A_{j}, B_{\ell}\right)>\pi\left(A_{k}, B_{\ell}\right)$, for any $B_{\ell}$, we say that $A_{j}$ is strictly dominant over $A_{k}$.

Based on the definition of dominant strategy above, we see from (17) that one of Alice's strategies strictly dominates the other whenever $\cos \theta_{B}>\cos \theta_{A} \cos \theta_{B}$, regardless of initial state. From Bob's point of view, the dominance of strategies arises whenever $\cos \theta_{A}>\cos \theta_{A} \cos \theta_{B}$. Since the classical games of "even or odd" and/or "heads or tails" do not have dominant strategies, we conclude that the game of a single qubit, which we are considering in this work, will only reproduce this classical situation when we set $\pi / 2<\theta_{\ell} \leq \pi$. Therefore, to extend the classical game to the quantum domain, we must assume that the value of $\theta_{\ell}$ is restricted to the half open interval $(\pi / 2, \pi]$.

A prevision of a player's average payment can be obtained whenever we consider that the game is played over and over again. In this situation, players must use each of the strategies in a random way and with a certain probability (solution by mixed strategies). To determine the best choice for each of the players, we must keep in mind that the optimal choice arises when a player's average gains, for each of the strategies his opponent uses, becomes the same [52,53]. When this is achieved, we say that the game has been solved or that the game has reached its equilibrium. This balance ensures that there is nothing your opponent can do to make your winnings less, as we will see later. Now we will determine the optimal strategies for our game in question, where these concepts can be better understood.

Consider that Alice plays randomly the strategies $\mathbb{1}$ and $\sigma_{x}$ with probabilities $\lambda$ and $1-\lambda$, respectively. With these considerations, we see that Alice's average payment will be $\Pi_{A}(\mathbb{1})$ when Bob uses the strategy $\mathbb{1}$ and $\Pi_{A}(\mathbf{x})$ when Bob uses the strategy $\sigma_{x}$, where we define

$$
\begin{aligned}
\Pi_{A}(\mathbb{1}) & =\lambda \pi_{+}(\mathbb{1}, \mathbb{1})+(1-\lambda) \pi_{+}(\mathbf{x}, \mathbb{1}) \\
\Pi_{A}(\mathbf{x}) & =\lambda \pi_{+}(\mathbb{1}, \mathbf{x})+(1-\lambda) \pi_{+}(\mathbf{x}, \mathbf{x}) .
\end{aligned}
$$

The optimal value of $\lambda$ arises when we impose $\Pi_{A}(\mathbb{1})=\Pi_{A}(\mathbf{x})$, obtaining

$$
\lambda=\frac{\left|\cos \theta_{A}\right|}{1+\left|\cos \theta_{A}\right|} \equiv \lambda_{o} .
$$


Note that in practice this adjusting is only possible for Alice if she has information about the parameter $\theta_{A}$ of her controlled adiabatic evolution.

From Bob's point of view, his optimal mixed strategy can be determined by considering that strategies $\mathbb{1}$ and $\sigma_{x}$ are randomly used with probability $\mu$ and $1-\mu$, respectively. Defining the payments

$$
\begin{aligned}
& \Pi_{B}(\mathbb{1})=-\mu \pi_{+}(\mathbb{1}, \mathbb{1})-(1-\mu) \pi_{+}(\mathbb{1}, \mathbf{x}) \\
& \Pi_{B}(\mathbf{x})=-\mu \pi_{+}(\mathbf{x}, \mathbb{1})-(1-\mu) \pi_{+}(\mathbf{x}, \mathbf{x}),
\end{aligned}
$$

the average gains of Bob will then be $\Pi_{B}(\mathbb{1})$, when Alice chooses to play $\mathbb{1}$, and $\Pi_{B}(\mathbf{x})$, when she plays $\sigma_{x}$. Now we can impose $\Pi_{B}(\mathbb{1})=\Pi_{B}(\mathbf{x})$ to get the optimal value

$$
\mu=\frac{\left|\cos \theta_{B}\right|}{1+\left|\cos \theta_{B}\right|} \equiv \mu_{o} .
$$

Again we see that Bob's best fit implies that he has knowledge of the parameter $\theta_{B}$ of his controlled adiabatic evolution. With these adjustments, the average gains of each player (replacing the optimal values of $\lambda$ and $\mu$ in (18) and (20), respectively) become

$$
\text { Alice: } \frac{x_{0}-x_{1}}{2} \$ ; \quad \text { Bob }: \frac{x_{1}-x_{0}}{2} \$ \text {. }
$$

Observe from (22) that Alice will have advantage if $x_{0}>x_{1}$ and disadvantage when $x_{1}>x_{0}$. When $x_{0}=x_{1}$, the average gains of each player become equal to zero and, therefore, the game becomes fair. The same conclusion is obtained if the initial game state is $|1\rangle$. Another important observation is that the average gains of each player are independent of the parameters $\theta_{A}$ and $\theta_{B}$, which means that the controlled adiabatic evolution does not interfere in the gains of each ones of players.

The role of optimal strategies can be understood in a simple way, as we see next. If Alice chooses the strategy $\mathbb{1}$, her average gain will be obtained by the weighted average of the gains according to each Bob strategy, that is, $-\Pi_{B}(\mathbb{1})$. If Alice chooses the strategy $\sigma_{x}$, her average gain becomes $-\Pi_{B}(\mathbf{x})$. Therefore, the net average gain of Alice, in the non-optimal situation where $\lambda \neq \lambda_{o}$, will be

$$
\Pi_{A}=-\lambda \Pi_{B}(\mathbb{1})-(1-\lambda) \Pi_{B}(\mathbf{x}) .
$$

If Bob uses his optimal strategy, then $\Pi_{A}=-\Pi_{B}(\mathbb{1})=-\Pi_{B}(\mathbf{x})=\left[\left(x_{0}-x_{1}\right) / 2\right] \$$, a result identical to Alice's payments in the optimal situation, when $\lambda=\lambda_{o}$. However, this scenario changes if Bob finds out that Alice is not making use of her optimal strategy by analyzing the statistical information of the strategies used by her, in the case of this information is available to players. To understand this point it, is appropriate to explicitly rewrite the average gains of Alice $\Pi_{A}$ in the form

$$
\begin{aligned}
\Pi_{A}= & {\left[\frac{x_{0}-x_{1}}{2}+\frac{x_{0}+x_{1}}{2}\left(1+\left|\cos \theta_{B}\right|\right)\left(1+\left|\cos \theta_{A}\right|\right)\right.} \\
& \left.\times\left(\mu-\mu_{o}\right)\left(\lambda-\lambda_{o}\right)\right] \$ .
\end{aligned}
$$


Note that if Alice inadvertently uses $\lambda$, such that

$$
0 \leq \lambda<\lambda_{o} \text { or } \lambda_{o}<\lambda \leq 1
$$

that Bob can adjust $\mu$ conveniently in the form

$$
\mu_{o}<\mu \leq 1 \text { or } 0 \leq \mu<\mu_{o}
$$

so that Alice's gain is lower and therefore his payments are higher. In other words, it is more safe for Alice to use the optimal strategy, because in this situation there is no way for Bob to modify his strategy to obtain a bigger gain and thus the equilibrium of the game arises.

\subsubsection{Inserting quantum strategies in adiabatic classic game}

In this section, we will analyze the game of the previous section with the inclusion of the quantum strategy $\mathbf{U}$. Using (15), we can define the payments (in units of \$)

$$
\begin{aligned}
\pi_{0}(\mathbb{1}, \mathbf{U})= & \frac{x_{0}-x_{1}}{2}+\frac{x_{0}+x_{1}}{2} \Phi_{B} \\
\pi_{0}(\mathbf{x}, \mathbf{U})= & \frac{x_{0}-x_{1}}{2}-\frac{x_{0}+x_{1}}{2} \Phi_{B}\left|\cos \theta_{A}\right| \\
\pi_{0}(\mathbf{U}, \mathbb{1})= & \frac{x_{0}-x_{1}}{2}+\frac{x_{0}+x_{1}}{2} \Phi_{A} \\
\pi_{0}(\mathbf{U}, \mathbf{x})= & \frac{x_{0}-x_{1}}{2}-\frac{x_{0}+x_{1}}{2} \Phi_{A}\left|\cos \theta_{B}\right| \\
\pi_{0}(\mathbf{U}, \mathbf{U})= & \frac{x_{0}-x_{1}}{2}+\frac{x_{0}+x_{1}}{2}\left[\Phi_{A} \Phi_{B}\right. \\
& \left.+\sin \phi_{A} \sin \phi_{B} \sin ^{2}\left(\frac{\theta_{A}}{2}\right) \sin ^{2}\left(\frac{\theta_{B}}{2}\right) \cos \delta_{B}\right],
\end{aligned}
$$

\begin{tabular}{|c|c|c|c|c|}
\hline$|\psi(0)\rangle=|0\rangle$ & Bob & & & \\
\hline \multirow[t]{4}{*}{ Alice } & Strategies & $\mathbb{1}$ & $\sigma_{x}$ & $\mathbf{U}$ \\
\hline & $\mathbb{1}$ & $\pi_{+}(\mathbb{1}, \mathbb{1})$ & $\pi_{+}(\mathbb{1}, \mathbf{x})$ & $\pi_{0}(\mathbb{1}, \mathbf{U})$ \\
\hline & $\sigma_{x}$ & $\pi_{+}(\mathbf{x}, \mathbb{1})$ & $\pi_{+}(\mathbf{x}, \mathbf{x})$ & $\pi_{0}(\mathbf{x}, \mathbf{U})$ \\
\hline & $\mathbf{U}$ & $\pi_{0}(\mathbf{U}, \mathbb{1})$ & $\pi_{0}(\mathbf{U}, \mathbf{x})$ & $\pi_{0}(\mathbf{U}, \mathbf{U})$. \\
\hline
\end{tabular}

and thus extend Tables 1, 2 and 3, which takes into account the quantum strategies, which is presented in Table 3 .

To find the extension of Table 2 for the situation involving quantum strategies, simply make the mutual exchange $x_{0} \rightarrow-x_{1}$ and $x_{1} \rightarrow-x_{0}$ in Table 3 .

Table 3 Extending Alice's payments in Table 1 for the situation involving quantum strategies when the initial game state is $|0\rangle$ 
Let us now turn to the analysis of the game. Since $-\left|\cos \theta_{A}\right| \leq \Phi_{A} \leq 1$, it is easy to verify that

$$
\begin{aligned}
& \pi_{0}(\mathbf{U}, \mathbb{1}) \leq \pi_{+}(\mathbb{1}, \mathbb{1}) \quad \text { and } \quad \pi_{+}(\mathbb{1}, \mathbf{x}) \leq \pi_{0}(\mathbf{U}, \mathbf{x}) \\
& \pi_{+}(\mathbf{x}, \mathbb{1}) \leq \pi_{0}(\mathbf{U}, \mathbb{1}) \quad \text { and } \quad \pi_{0}(\mathbf{U}, \mathbf{x}) \leq \pi_{+}(\mathbf{x}, \mathbf{x})
\end{aligned}
$$

and therefore there is no (weak) dominance of $\mathbf{U}$ over the classical strategies $\mathbb{1}$ and $\sigma_{x}$, respectively. It is important to note that the strategy $\mathbf{U}$ can represent one or a continuum of strategies depending on whether the parameter $\phi_{A}$ and $\phi_{B}$ are fixed or vary. As they are parameters of possession of each player, it is reasonable to conclude that $\mathbf{U}$ represents a set of strategies, remembering that $\theta_{A}$ and $\theta_{B}$ are fixed parameters during all games. Despite this, in our analysis to obtain the optimal strategy, we will assume that $\mathbf{U}$ represents a single strategy, without loss of generality, as will become clear below.

Defining the payments

$$
\begin{aligned}
& \Pi_{A}(\mathbb{1})=\lambda_{1} \pi_{+}(\mathbb{1}, \mathbb{1})+\lambda_{2} \pi_{+}(\mathbf{x}, \mathbb{1})+\lambda_{3} \pi(\mathbf{U}, \mathbb{1}) \\
& \Pi_{A}(\mathbf{x})=\lambda_{1} \pi_{+}(\mathbb{1}, \mathbf{x})+\lambda_{2} \pi_{+}(\mathbf{x}, \mathbf{x})+\lambda_{3} \pi(\mathbf{U}, \mathbf{x}) \\
& \Pi_{A}(\mathbf{U})=\lambda_{1} \pi(\mathbb{1}, \mathbf{U})+\lambda_{2} \pi(\mathbf{x}, \mathbf{U})+\lambda_{3} \pi(\mathbf{U}, \mathbf{U}),
\end{aligned}
$$

and assuming that Alice uses the classical strategy $\mathbb{1}\left(\sigma_{x}\right)$ with probability $\lambda_{1}\left(\lambda_{2}\right)$ and the quantum strategy $\mathbf{U}$ with probability $\lambda_{3}=1-\lambda_{1}-\lambda_{2}$, it can be verified that the average gains of Alice become $\Pi_{A}(\mathbb{1}), \Pi_{A}(\mathbf{x})$ and $\Pi_{A}(\mathbf{U})$ if Bob uses the strategy $\mathbb{1}$, $\sigma_{x}$ and $\mathbf{U}$, respectively. The optimal strategy arises when $\Pi_{A}(\mathbb{1})=\Pi_{A}(\mathbf{x})=\Pi_{A}(\mathbf{U})$, in which case we obtain the relations

$$
\begin{gathered}
\lambda_{1}-\frac{\left|\cos \theta_{A}\right|}{1+\left|\cos \theta_{A}\right|}-\left(\lambda_{1}+\lambda_{2}-1\right) \cos ^{2}\left(\phi_{A} / 2\right)=0 \\
\left(\lambda_{1}+\lambda_{2}-1\right) \sin \phi_{A} \sin ^{2}\left(\theta_{B} / 2\right) \sin \phi_{B} \cos \delta_{B}=0 .
\end{gathered}
$$

Note from (31) that Alice's optimal strategy setting involves Bob's parameters $\left(\phi_{B}, \theta_{B}\right.$ and $\delta_{B}$ ) that we assume that be unknown to Alice. Therefore, it is reasonable to assume that the best fit for Alice is obtained so that it is independent of these parameters. Thus, the relation (31) leads us to three options:

(i) In the first option, we can set $\phi_{A}=0$ and thus we must set $\lambda_{2}$, such that

$$
\lambda_{2}=\frac{1}{1+\left|\cos \theta_{A}\right|},
$$

leaving free $\lambda_{1}$

(ii) In the second option, we can adjust $\phi_{A}=\pi$ and, consequently, we obtain an adjustment for $\lambda_{1}$ given by 


$$
\lambda_{1}=\frac{\left|\cos \theta_{A}\right|}{1+\left|\cos \theta_{A}\right|},
$$

while we can choose any value for $\lambda_{2}$;

(iii) Finally, the third option is to set $\lambda_{1}+\lambda_{2}=1$ (discarding the quantum strategies in the game), in which case we retrieve the results at (32) and (33). It is important to mention that this case can be seen as a particular case of (i) and also of (ii), since in both cases one of the $\lambda$ 's have a free value.

For any of the three situations defined above Alice's earnings are independent of Bob's action and become the same as in the classical case (22). This result leads us to the conclusion that quantum strategies can be neglected.

It is important to note that in the situation where Bob is only a classical player, the condition (31b) is not required. However, Alice's average payment will still be given by Eq. (22), as can be concluded by using (31a) in (30a), regardless of $\phi_{A}$ and $\theta_{A}$. Therefore, we see that quantum strategies can still be ignored in the process of obtaining the optimal strategy, even in the case where Alice confronts a classical player, when the initial state is $|0\rangle$ or $|1\rangle$.

\subsubsection{Adiabatic game with a initial superposition state}

We will now analyze the situation where the initial game state becomes a superposition and thus determine the regimes in which a quantum player obtains advantages over a classical player.

Using Eq. (15), we can verify that payments, in units of \$, for each combination of strategies become

$$
\begin{aligned}
\pi(\mathbb{1}, \mathbb{1})= & \frac{x_{0}-x_{1}}{2}+\frac{x_{0}+x_{1}}{2}\left(\left|\alpha_{0}\right|^{2}-\left|\alpha_{1}\right|^{2}\right) \\
\pi(\mathbb{1}, \mathbf{x})= & \frac{x_{0}-x_{1}}{2}-\frac{x_{0}+x_{1}}{2}\left(\left|\alpha_{0}\right|^{2}-\left|\alpha_{1}\right|^{2}\right)\left|\cos \theta_{B}\right| \\
\pi(\mathbb{1}, \mathbf{U})= & \frac{x_{0}-x_{1}}{2}+\frac{x_{0}+x_{1}}{2}\left[\left(\left|\alpha_{0}\right|^{2}-\left|\alpha_{1}\right|^{2}\right) \Phi_{B}\right. \\
& \left.+2 \operatorname{Re}\left(\alpha_{0} \alpha_{1}^{*}\right) \sin ^{2}\left(\theta_{B} / 2\right) \sin \phi_{B}\right] \\
\pi(\mathbf{x}, \mathbb{1})= & \frac{x_{0}-x_{1}}{2}-\frac{x_{0}+x_{1}}{2}\left(\left|\alpha_{0}\right|^{2}-\left|\alpha_{1}\right|^{2}\right)\left|\cos \theta_{A}\right| \\
\pi(\mathbf{x}, \mathbf{x})= & \frac{x_{0}-x_{1}}{2}+\frac{x_{0}+x_{1}}{2}\left(\left|\alpha_{0}\right|^{2}-\left|\alpha_{1}\right|^{2}\right)\left|\cos \theta_{A} \cos \theta_{B}\right| \\
\pi(\mathbf{x}, \mathbf{U})= & \frac{x_{0}-x_{1}}{2}-\frac{x_{0}+x_{1}}{2}\left[\left(\left|\alpha_{0}\right|^{2}-\left|\alpha_{1}\right|^{2}\right)\left|\cos \theta_{A}\right| \Phi_{B}\right. \\
& \left.+2 \operatorname{Re}\left(\alpha_{0} \alpha_{1}^{*}\right) \sin ^{2}\left(\theta_{B} / 2\right) \sin \phi_{B}\left|\cos \theta_{A}\right|\right] \\
\pi(\mathbf{U}, \mathbb{1})= & \frac{x_{0}-x_{1}}{2}+\frac{x_{0}+x_{1}}{2}\left[\left(\left|\alpha_{0}\right|^{2}-\left|\alpha_{1}\right|^{2}\right) \Phi_{A}\right. \\
& \left.+2 \operatorname{Re}\left(\alpha_{0} \alpha_{1}^{*}\right) \sin ^{2}\left(\theta_{A} / 2\right) \sin \phi_{A}\right]
\end{aligned}
$$




$$
\begin{aligned}
\pi(\mathbf{U}, \mathbf{x})= & \frac{x_{0}-x_{1}}{2}-\frac{x_{0}+x_{1}}{2}\left[\left(\left|\alpha_{0}\right|^{2}-\left|\alpha_{1}\right|^{2}\right)\left|\cos \theta_{B}\right| \Phi_{A}\right. \\
& \left.-2 \operatorname{Re}\left(\alpha_{0} \alpha_{1}^{*}\right) \sin ^{2}\left(\theta_{A} / 2\right) \sin \phi_{A}\right] \\
\pi(\mathbf{U}, \mathbf{U})= & \frac{x_{0}-x_{1}}{2}+\frac{x_{0}+x_{1}}{2}\left\{( | \alpha _ { 0 } | ^ { 2 } - | \alpha _ { 1 } | ^ { 2 } ) \left[\Phi_{A} \Phi_{B}\right.\right. \\
& \left.+\sin ^{2}\left(\theta_{A} / 2\right) \sin ^{2}\left(\theta_{B} / 2\right) \sin \phi_{A} \sin \phi_{B} \cos \delta_{B}\right] \\
& -2 \operatorname{Im}\left(\alpha_{0} \alpha_{1}^{*}\right) \sin ^{2}\left(\theta_{A} / 2\right) \sin ^{2}\left(\theta_{B} / 2\right) \sin \phi_{A} \sin \delta_{B} \\
& +2 \operatorname{Re}\left(\alpha_{0} \alpha_{1}^{*}\right)\left[\Phi_{A} \sin ^{2}\left(\theta_{B} / 2\right) \sin \phi_{B}\right. \\
& +\left(1-\sin ^{2}\left(\theta_{B} / 2\right)\left[1+\cos \phi_{B} \cos \delta_{B}\right]\right) \\
& \left.\left.\times \sin ^{2}\left(\theta_{A} / 2\right) \sin \phi_{A}\right]\right\}
\end{aligned}
$$

From the payments defined above, it is crucial to note the lack of symmetry with respect to payments $\pi(\mathbf{x}, \mathbf{U})$ and $\pi(\mathbf{U}, \mathbf{x})$. So far, in this work, all the non-diagonal elements were symmetrical with respect to the permutation of the $A \leftrightarrow B$ indices was performed. This breaking of symmetry, which arises when $\alpha_{0}$ and $\alpha_{1}$ are nonzero, allows a differentiation between the players with respect to the order in which each one applies their strategy, as we will see below. This fact leads us to think that, in the presence of symmetry, all players are equivalent and therefore no one should get benefits. On the other hand, the absence of symmetry suggests that quantum strategies may play a relevant role, as we will see below.

\subsection{Regimes of dominance}

Let us now analyze in what regimes of parameters that the quantum player Alice possesses dominant strategies with respect to a classic player Bob. It is easy to show that there is no dominance between the classical strategies of Alice, since in the situation where $\pi(\mathbb{1}, \mathbb{1})$ is smaller (greater) than $\pi(\mathbf{x}, \mathbb{1})$ it is verified that $\pi(\mathbb{1}, \mathbf{x})$ is larger (smaller) than $\pi(\mathbf{x}, \mathbf{x})$ and hence independently of any relation between $\pi(\mathbb{1}, \mathbf{U})$ and $\pi(\mathbf{x}, \mathbf{U})$, we conclude that $\mathbb{1}$ does not dominate $\sigma_{x}$ and vice versa. In addition, since the phases $\delta_{A}$ play no role in (34), we can discard the Alice quantum strategies involved in cases where $\phi_{A}=0, \pi$.

Domain of $\mathbf{U}$ over $\mathbb{1}$. For Alice's quantum strategy $\mathbf{U}$ to dominate (weakly) the strategy $\mathbb{1}$ it is necessary that

$$
\pi(\mathbf{U}, \mathbb{1}) \geq \pi(\mathbb{1}, \mathbb{1}) \text { and } \pi(\mathbf{U}, \mathbf{x}) \geq \pi(\mathbb{1}, \mathbf{x}) .
$$

These inequalities imply, respectively, in the relations

$$
\begin{aligned}
& 2 \operatorname{Re}\left(\alpha_{0} \alpha_{1}^{*}\right) \geq\left(\left|\alpha_{0}\right|^{2}-\left|\alpha_{1}\right|^{2}\right) \tan \left(\phi_{A} / 2\right) \\
& 2 \operatorname{Re}\left(\alpha_{0} \alpha_{1}^{*}\right) \geq-\left(\left|\alpha_{0}\right|^{2}-\left|\alpha_{1}\right|^{2}\right) \tan \left(\phi_{A} / 2\right)\left|\cos \theta_{B}\right| .
\end{aligned}
$$


However, as $0<\phi_{A}<\pi$, we note that $\tan \left(\phi_{A} / 2\right)>0$ and therefore there is no dominance for the case where $\operatorname{Re}\left(\alpha_{0} \alpha_{1}^{*}\right)<0$, regardless of the signal of $\left|\alpha_{0}\right|^{2}-\left|\alpha_{1}\right|^{2}$, since one of the two inequalities in (36) must be violated. If $\operatorname{Re}\left(\alpha_{0} \alpha_{1}^{*}\right)=0$, we note that Eq. (36) can be satisfied only for the particular situation where $\left|\alpha_{0}\right|=\left|\alpha_{1}\right|$. In the most comprehensive case, where the initial state is such that $\operatorname{Re}\left(\alpha_{0} \alpha_{1}^{*}\right)>0$, we have three possibilities:

(i) When $\left|\alpha_{0}\right|>\left|\alpha_{1}\right|$, the Alice's quantum strategy $\mathbf{U}$ dominates $\mathbb{1}$ whenever

$$
0<\phi_{A} \leq 2 \arctan \Lambda_{\alpha}
$$

where we define

$$
\Lambda_{\alpha}=\left|\frac{2 \operatorname{Re}\left(\alpha_{0} \alpha_{1}^{*}\right)}{\left|\alpha_{0}\right|^{2}-\left|\alpha_{1}\right|^{2}}\right| .
$$

(ii) If $\left|\alpha_{0}\right|<\left|\alpha_{1}\right|$, the dominance over classical strategy $\mathbb{1}$ arises when

$$
0<\phi_{A} \leq 2 \arctan \left(\Lambda_{\alpha} /\left|\cos \theta_{B}\right|\right)
$$

(iii) For $\left|\alpha_{0}\right|=\left|\alpha_{1}\right|$, the quantum strategy $\mathbf{U}$ always dominates $\mathbb{1}$ for any allowed value of $\phi_{A}$, that is, $0<\phi_{A}<\pi$.

Domain of $\mathbf{U}$ over $\sigma_{x}$. The Alice's quantum strategy $\mathbf{U}$ will dominate the classical strategy $\sigma_{x}$ whenever

$$
\pi(\mathbf{U}, \mathbb{1}) \geq \pi(\mathbf{x}, \mathbb{1}) \text { and } \pi(\mathbf{U}, \mathbf{x}) \geq \pi(\mathbf{x}, \mathbf{x})
$$

which imply, respectively, in the inequalities

$$
\begin{aligned}
& 2 \operatorname{Re}\left(\alpha_{0} \alpha_{1}^{*}\right) \tan \left(\phi_{A} / 2\right) \geq-\left(\left|\alpha_{0}\right|^{2}-\left|\alpha_{1}\right|^{2}\right) \\
& 2 \operatorname{Re}\left(\alpha_{0} \alpha_{1}^{*}\right) \tan \left(\phi_{A} / 2\right) \geq\left(\left|\alpha_{0}\right|^{2}-\left|\alpha_{1}\right|^{2}\right)\left|\cos \theta_{B}\right| .
\end{aligned}
$$

Again we observe from (41) that there are no solutions for the case where $\operatorname{Re}\left(\alpha_{0} \alpha_{1}^{*}\right)<$ 0 and for the case where $\operatorname{Re}\left(\alpha_{0} \alpha_{1}^{*}\right)=0$ the only solution is obtained for the particular situation where $\left|\alpha_{0}\right|=\left|\alpha_{1}\right|$. In the case of interest, where $\operatorname{Re}\left(\alpha_{0} \alpha_{1}^{*}\right)>0$, we have the following solutions:

(i) If $\left|\alpha_{0}\right|>\left|\alpha_{1}\right|$, the Alice's quantum strategy $\mathbf{U}$ dominates $\sigma_{x}$ whenever

$$
2 \arctan \left(\left|\cos \theta_{B}\right| / \Lambda_{\alpha}\right) \leq \phi_{A}<\pi
$$

(ii) When $\left|\alpha_{0}\right|<\left|\alpha_{1}\right|$, the inequality obtained when we impose the domain of $\mathbf{U}$ over $\sigma_{x}$ becomes

$$
2 \arctan \left(1 / \Lambda_{\alpha}\right) \leq \phi_{A}<\pi
$$

(iii) For $\left|\alpha_{0}\right|=\left|\alpha_{1}\right|$, the quantum strategy $\mathbf{U}$ always dominates $\sigma_{x}$ for any allowed value of $\phi_{A}$, that is, $0<\phi_{A}<\pi$. 
Mutual dominance of $\mathbf{U}$ over $\mathbb{1}$ and $\sigma_{x}$. According to each regime seen above, the intersection interval represents the mutual domain of Alice's quantum strategy $\mathbf{U}$ over the classical strategies $\mathbb{1}$ and $\sigma_{x}$. Note, therefore, that this interval is defined by:

$$
\begin{aligned}
2 \arctan \left(\frac{\left|\cos \theta_{B}\right|}{\Lambda_{\alpha}}\right) & \leq \phi_{A} \leq 2 \arctan \Lambda_{\alpha}, \quad \text { if }\left|\alpha_{0}\right|>\left|\alpha_{1}\right| \\
2 \arctan \left(\Lambda_{\alpha}^{-1}\right) & \leq \phi_{A} \leq 2 \arctan \left(\frac{\Lambda_{\alpha}}{\left|\cos \theta_{B}\right|}\right), \quad \text { if }\left|\alpha_{0}\right|<\left|\alpha_{1}\right|,
\end{aligned}
$$

provided that the inequality $\left|\cos \theta_{B}\right| \leq \Lambda_{\alpha}^{2}$ be verified. It is interesting to remember that when $\left|\alpha_{0}\right| \rightarrow\left|\alpha_{1}\right|$, that $\Lambda_{\alpha} \rightarrow \infty$ and therefore, both inequalities in Eq. (44) become the interval $0<\phi_{A}<\pi$.

Absence of dominance. Excluding the situation where $\operatorname{Re}\left(\alpha_{0} \alpha_{1}^{*}\right)<0$, for the case where $\operatorname{Re}\left(\alpha_{0} \alpha_{1}^{*}\right)>0$ there will be non-dominance regimes defined by the intervals:

$$
\begin{aligned}
2 \arctan \Lambda_{\alpha}<\phi_{A}<2 \arctan \left(\frac{\left|\cos \theta_{B}\right|}{\Lambda_{\alpha}}\right), \quad \text { if }\left|\alpha_{0}\right|>\left|\alpha_{1}\right| \\
2 \arctan \left(\frac{\Lambda_{\alpha}}{\left|\cos \theta_{B}\right|}\right)<\phi_{A}<2 \arctan \left(\Lambda_{\alpha}^{-1}\right), \quad \text { if }\left|\alpha_{0}\right|<\left|\alpha_{1}\right|,
\end{aligned}
$$

where it is assumed that the inequality $\left|\cos \theta_{B}\right|>\Lambda_{\alpha}^{2}$ be satisfied. Note that $\left|\alpha_{0}\right| \rightarrow 0$ or $\left|\alpha_{1}\right| \rightarrow 0$ implies in $\Lambda_{\alpha} \rightarrow 0$ and therefore Eq. (45) become the interval $0<\phi_{A}<$ $\pi$.

\subsection{Quantum player payments}

The optimal payout of each player is associated with the use of optimal strategies for both players. To obtain these values, we still have to determine the dominating strategies from Bob's point of view. Before proceeding with this task, it is important to note that whenever one strategy is dominated by another, it can be discarded without modified the game result (called equilibrium). When we discard a strategy in the game analysis by a player, we must remake the analysis for the previous player and see if some dominant strategy emerges in this reduced strategy space.

Let us now determine Alice's payment in units of $\$$, separating our analysis into two regimes that depend on the relationships between the initial state parameters $\left|\alpha_{0}\right|$ and $\left|\alpha_{1}\right|$.

Regime in which $\left|\alpha_{0}\right| \geq\left|\alpha_{1}\right|$. In this regime we will analyze the game solutions for certain ranges of $\phi_{A}$.

(i) For the situation where initially the Alice's quantum strategy $\mathbf{U}$ dominates only $\mathbb{1}$, defined by the interval $0<\phi_{A} \leq 2 \arctan \left(\min \left\{\Lambda_{\alpha},\left|\cos \theta_{B}\right| / \Lambda_{\alpha}\right\}\right)$, the game solution depends on the analysis of Bob's strategies:

(a) The Bob's classical strategy $\mathbb{1}$ dominates $\sigma_{x}$, that is,

$$
\pi(\mathbf{x}, \mathbf{x}) \geq \pi(\mathbf{x}, \mathbb{1}) \text { and } \pi(\mathbf{U}, \mathbf{x}) \geq \pi(\mathbf{U}, \mathbb{1}),
$$


which occurs whenever $\phi_{c} \leq \phi_{A} \leq 2 \arctan \left(\min \left\{\Lambda_{\alpha},\left|\cos \theta_{B}\right| / \Lambda_{\alpha}\right\}\right)$, where we define the critical parameter

$$
\phi_{c}=2 \arcsin \left\{1 /\left[\sqrt{2} \sin \left(\theta_{A} / 2\right)\right]\right\} \text {. }
$$

In this situation, discarding Bob's strategy $\sigma_{x}$, it is verified that the Alice's quantum strategy $\mathbf{U}$ starts to dominate $\sigma_{x}$, because in this reduced space of strategies, we have that $\pi(\mathbf{U}, \mathbb{1}) \geq \pi(\mathbf{x}, \mathbb{1})$, according to (41a). Therefore, the game solution is obtained with Alice by applying $\mathbf{U}$ and Bob $\mathbb{1}$, leading to a payment to Alice given by

$$
\begin{aligned}
\Pi_{A}= & \pi(\mathbf{U}, \mathbb{1})=\frac{x_{0}-x_{1}}{2}+\left.\frac{x_{0}+x_{1}}{2}|| \alpha_{0}\right|^{2}-\left|\alpha_{1}\right|^{2} \mid \\
& \times\left\{\sin \phi_{A} \sin ^{2}\left(\theta_{A} / 2\right)\left[\Lambda_{\alpha}-\tan \left(\phi_{A} / 2\right)\right]+1\right\} .
\end{aligned}
$$

(b) When there is no dominance of any Bob's classical strategy, situation in which $\phi_{A} \leq 2 \arctan \left(\min \left\{\Lambda_{\alpha},\left|\cos \theta_{B}\right| / \Lambda_{\alpha}\right\}\right)<\phi_{c}$, the game solution is obtained by the application of mixed strategies. To obtain this solution, consider that $\lambda_{1}$ and $1-\lambda_{1}$ are the probabilities of Alice plays the strategy $\sigma_{x}$ and $\mathbf{U}$, respectively. Imposing the equality

$$
\lambda_{1} \pi(\mathbf{x}, \mathbb{1})+\left(1-\lambda_{1}\right) \pi(\mathbf{U}, \mathbb{1})=\lambda_{1} \pi(\mathbf{x}, \mathbf{x})+\left(1-\lambda_{1}\right) \pi(\mathbf{U}, \mathbf{x}),
$$

we obtain

$$
\begin{aligned}
\lambda_{1} & =\frac{\pi(\mathbf{U}, \mathbf{x})-\pi(\mathbf{U}, \mathbb{1})}{\pi(\mathbf{x}, \mathbb{1})-\pi(\mathbf{x}, \mathbf{x})+\pi(\mathbf{U}, \mathbf{x})-\pi(\mathbf{U}, \mathbb{1})} \\
& =\frac{1-2 \sin ^{2}\left(\theta_{A} / 2\right) \sin ^{2}\left(\phi_{A} / 2\right)}{2 \sin ^{2}\left(\theta_{A} / 2\right) \cos ^{2}\left(\phi_{A} / 2\right)}>0,
\end{aligned}
$$

and Alice's payment becomes

$$
\begin{aligned}
\Pi_{A}= & \pi(\operatorname{mix} 1)=\frac{x_{0}-x_{1}}{2}+\left(x_{0}+x_{1}\right) \\
& \times\left|\operatorname{Re}\left(\alpha_{0} \alpha_{1}^{*}\right)\right| \tan \left(\phi_{A} / 2\right)\left|\cos \left(\theta_{A}\right)\right| .
\end{aligned}
$$

(ii) Consider that Alice's quantum strategy $\mathbf{U}$ dominates both the classical strategies $\mathbb{1}$ and $\sigma_{x}$, defined by the interval $2 \arctan \left(\left|\cos \theta_{B}\right| / \Lambda_{\alpha}\right) \leq \phi_{A} \leq 2 \arctan \Lambda_{\alpha}$, presented in (44a). Analyzing Bob's strategies, we find that two situations can occur:

(a) When Bob's classical strategy $\sigma_{x}$ dominates $\mathbb{1}$, in which case 2 arctan $\left(\left|\cos \theta_{B}\right| / \Lambda_{\alpha}\right) \leq \phi_{A} \leq \phi_{c}$, the game solution is obtained with Alice by applying the strategy $\mathbf{U}$ and Bob $\sigma_{x}$, resulting in a payment to Alice of

$$
\Pi_{A}=\pi(\mathbf{U}, \mathbf{x})=\frac{x_{0}-x_{1}}{2}+\left.\frac{x_{0}+x_{1}}{2}|| \alpha_{0}\right|^{2}-\left|\alpha_{1}\right|^{2} \mid
$$




$$
\times\left\{\sin \phi_{A} \sin ^{2}\left(\theta_{A} / 2\right)\left[\Lambda_{\alpha}+\tan \left(\phi_{A} / 2\right)\left|\cos \theta_{B}\right|\right]-\left|\cos \theta_{B}\right|\right\}
$$

(b) In the opposite situation to (i), if Bob's classical strategy $\mathbb{1}$ dominates $\sigma_{x}$, in which case $\phi_{c} \leq \phi_{A} \leq 2 \arctan \Lambda_{\alpha}$, the game solution is obtained by Alice choosing $\mathbf{U}$ and Bob $\mathbb{1}$, resulting in the payment to Alice given by (48).

(iii) Let us consider now Alice's quantum strategy $\mathbf{U}$ initially dominates only $\sigma_{x}$, defined by the interval $2 \arctan \left(\max \left\{\Lambda_{\alpha},\left|\cos \theta_{B}\right| / \Lambda_{\alpha}\right\}\right) \leq \phi_{A}<\pi$. For this case there is a solution for each domain regime of Bob's strategies:

(a) When Bob's classical strategy $\sigma_{x}$ dominates $\mathbb{1}$, that is,

$$
\pi(\mathbb{1}, \mathbf{x}) \leq \pi(\mathbb{1}, \mathbb{1}) \text { and } \pi(\mathbf{U}, \mathbf{x}) \leq \pi(\mathbf{U}, \mathbb{1})
$$

which is satisfied for the range defined by $2 \arctan \left(\max \left\{\Lambda_{\alpha},\left|\cos \theta_{B}\right| / \Lambda_{\alpha}\right\}\right) \leq$ $\phi_{A} \leq \phi_{c}$. Disregarding Bob's strategy $\mathbb{1}$, it turns out that Alice's quantum strategy $\mathbf{U}$ dominates $\mathbb{1}$, because in this reduced strategy space we have $\pi(\mathbf{U}, \mathbf{x}) \geq \pi(\mathbb{1}, \mathbf{x})$, according to (36b). As a consequence, the game solution is obtained with Alice by applying $\mathbf{U}$ and Bob $\sigma_{x}$, leading to an Alice's payment given by (52).

(b) In case there is no dominance between Bob's classical strategies, which occurs in the range $\phi_{c}<2 \arctan \left(\max \left\{\Lambda_{\alpha},\left|\cos \theta_{B}\right| / \Lambda_{\alpha}\right\}\right) \leq \phi_{A}$, the solution is again obtained by the application of mixed strategies. If $\lambda_{x}$ and $1-\lambda_{x}$ are the probabilities of Alice play $\mathbb{1}$ and $\mathbf{U}$, respectively, by imposing the equality

$$
\lambda_{x} \pi(\mathbb{1}, \mathbb{1})+\left(1-\lambda_{x}\right) \pi(\mathbf{U}, \mathbb{1})=\lambda_{x} \pi(\mathbb{1}, \mathbf{x})+\left(1-\lambda_{x}\right) \pi(\mathbf{U}, \mathbf{x}),
$$

we obtain

$$
\begin{aligned}
\lambda_{x} & =\frac{\pi(\mathbf{U}, \mathbf{x})-\pi(\mathbf{U}, \mathbb{1})}{\pi(\mathbb{1}, \mathbb{1})-\pi(\mathbb{1}, \mathbf{x})+\pi(\mathbf{U}, \mathbf{x})-\pi(\mathbf{U}, \mathbb{1})} \\
& =\frac{2 \sin ^{2}\left(\theta_{A} / 2\right) \sin ^{2}\left(\phi_{A} / 2\right)-1}{2 \sin ^{2}\left(\theta_{A} / 2\right) \sin ^{2}\left(\phi_{A} / 2\right)}>0,
\end{aligned}
$$

and Alice's payment becomes

$$
\Pi_{A}=\pi(\operatorname{mix} 2)=\frac{x_{0}-x_{1}}{2}+\left(x_{0}+x_{1}\right)\left|\operatorname{Re}\left(\alpha_{0} \alpha_{1}^{*}\right)\right| \cot \left(\phi_{A} / 2\right) .
$$

(iv) When there is no dominance between any of Alice's strategies, defined by the interval $2 \arctan \Lambda_{\alpha}<\phi_{A}<2 \arctan \left(\left|\cos \theta_{B}\right| / \Lambda_{\alpha}\right)$, and the same occurs for Bob, the game solution is obtained by the use of mixed strategies. Consider, therefore, that Alice chooses to play $\lambda_{1}^{\prime}\left(\lambda_{2}^{\prime}\right)$ times the classical strategy $\mathbb{1}\left(\sigma_{x}\right)$ and $1-\lambda_{1}^{\prime}-\lambda_{2}^{\prime}$ times the quantum strategy $\mathbf{U}$. Imposing the equality

$$
\begin{aligned}
& \lambda_{1}^{\prime} \pi(\mathbb{1}, \mathbb{1})+\lambda_{2}^{\prime} \pi(\mathbf{x}, \mathbb{1})+\left(1-\lambda_{1}^{\prime}-\lambda_{2}^{\prime}\right) \pi(\mathbf{U}, \mathbb{1}) \\
& =\lambda_{1}^{\prime} \pi(\mathbb{1}, \mathbf{x})+\lambda_{2}^{\prime} \pi(\mathbf{x}, \mathbf{x})+\left(1-\lambda_{1}^{\prime}-\lambda_{2}^{\prime}\right) \pi(\mathbf{U}, \mathbf{x}),
\end{aligned}
$$


we obtain the condition

$$
\lambda_{1}^{\prime}-\lambda_{2}^{\prime}\left|\cos \left(\theta_{A}\right)\right|+\left(1-\lambda_{1}^{\prime}-\lambda_{2}^{\prime}\right) \Phi_{A}=0
$$

The condition (58) requires that the game solution be different for different intervals of $\phi_{A}$ :

(a) For the interval defined by $2 \arctan \Lambda_{\alpha}<\phi_{A} \leq \phi_{c}$ we must choose

$$
\lambda_{1}^{\prime}=0 \quad \text { and } \quad \lambda_{2}^{\prime}=\frac{1-2 \sin ^{2}\left(\theta_{A} / 2\right) \sin ^{2}\left(\phi_{A} / 2\right)}{2 \sin ^{2}\left(\theta_{A} / 2\right) \cos ^{2}\left(\phi_{A} / 2\right)}
$$

so that Alice's payment becomes (51).

(b) In the interval $\phi_{c} \leq \phi_{A}<2 \arctan \left(\left|\cos \theta_{B}\right| / \Lambda_{\alpha}\right)$ we must choose

$$
\lambda_{2}^{\prime}=0 \quad \text { and } \quad \lambda_{1}^{\prime}=\frac{2 \sin ^{2}\left(\theta_{A} / 2\right) \sin ^{2}\left(\phi_{A} / 2\right)-1}{2 \sin ^{2}\left(\theta_{A} / 2\right) \sin ^{2}\left(\phi_{A} / 2\right)},
$$

which leads Alice to receive the payment set by (56).

Regime in which $\left|\alpha_{0}\right| \leq\left|\alpha_{1}\right|$. In the situation where $\left|\alpha_{0}\right| \leq\left|\alpha_{1}\right|$ we again have different game solutions, depending on the intervals at which $\phi_{A}$ varies.

(i) The first case to be analyzed will be the one in which Alice's quantum strategy $\mathbf{U}$ initially dominates only $\mathbb{1}$, defined by the interval $0<\phi_{A} \leq$ $2 \arctan \left(\min \left\{1 / \Lambda_{\alpha}, \Lambda_{\alpha} /\left|\cos \theta_{B}\right|\right\}\right)$. In this range of $\phi_{A}$ we can have two different solutions, depending on the dominance analysis of Bob's strategies:

(a) If the Bob's classical strategy $\sigma_{x}$ dominates $\mathbb{1}$, that is,

$$
\pi(\mathbf{x}, \mathbf{x}) \leq \pi(\mathbf{x}, \mathbb{1}) \text { and } \pi(\mathbf{U}, \mathbf{x}) \leq \pi(\mathbf{U}, \mathbb{1})
$$

which occurs whenever $\phi_{c} \leq \phi_{A} \leq 2 \arctan \left(\min \left\{1 / \Lambda_{\alpha}, \Lambda_{\alpha} /\left|\cos \theta_{B}\right|\right\}\right)$, we can discard strategy $\mathbb{1}$ in our analysis. In this situation, it is verified that Alice's quantum strategy $\mathbf{U}$ dominates $\sigma_{x}$, since in this reduced strategy space, we have $\pi(\mathbf{U}, \mathbf{x}) \geq \pi(\mathbf{x}, \mathbf{x})$, as obtained from (41b). Therefore, the game solution is obtained with Alice by applying $\mathbf{U}$ and Bob $\sigma_{x}$, leading to an Alice's payment given by

$$
\begin{aligned}
\Pi_{A}= & \pi(\mathbf{U}, \mathbf{x})=\frac{x_{0}-x_{1}}{2}+\left.\frac{x_{0}+x_{1}}{2}|| \alpha_{0}\right|^{2}-\left|\alpha_{1}\right|^{2} \mid \\
& \times\left\{\sin \phi_{A} \sin ^{2}\left(\theta_{A} / 2\right)\left[\Lambda_{\alpha}-\tan \left(\phi_{A} / 2\right)\left|\cos \theta_{B}\right|\right]+\left|\cos \theta_{B}\right|\right\} .
\end{aligned}
$$

(b) When there is no dominance between Bob's classical strategies, which occurs in the interval where $\phi_{c}>2 \arctan \left(\min \left\{1 / \Lambda_{\alpha}, \Lambda_{\alpha} /\left|\cos \theta_{B}\right|\right\}\right) \geq \phi_{A}$, the game solution is obtained by the application of mixed strategies, which is identical to the situation occurred in item (b) of (i) for the regime $\left|\alpha_{0}\right| \geq$ $\left|\alpha_{1}\right|$, analyzed above. Therefore, for this case we must adjust the probabilities according to (50), which leads Alice to receive a payment given by (51). 
(ii) For the situation where Alice's quantum strategy $\mathbf{U}$ dominates both classical strategies $\mathbb{1}$ and $\sigma_{x}$, defined by the interval $2 \arctan \left(1 / \Lambda_{\alpha}\right) \leq \phi_{A} \leq$ $2 \arctan \left(\Lambda_{\alpha} /\left|\cos \theta_{B}\right|\right)$, as we have seen in Eq. (44b), we can have two different solutions:

(a) If Bob's classical strategy $\mathbb{1}$ dominates $\sigma_{x}$, in which case $2 \arctan \left(1 / \Lambda_{\alpha}\right) \leq$ $\phi_{A} \leq \phi_{c}$, the game solution occurs for the situation where Alice plays $\mathbf{U}$ and Bob plays $\mathbb{1}$, leading to a payment to Alice equal to

$$
\begin{aligned}
\Pi_{A}= & \pi(\mathbf{U}, \mathbb{1})=\frac{x_{0}-x_{1}}{2}+\left.\frac{x_{0}+x_{1}}{2}|| \alpha_{0}\right|^{2}-\left|\alpha_{1}\right|^{2} \mid \\
& \times\left\{\sin \phi_{A} \sin ^{2}\left(\theta_{A} / 2\right)\left[\Lambda_{\alpha}+\tan \left(\phi_{A} / 2\right)\right]-1\right\} .
\end{aligned}
$$

(b) If Bob's classical strategy $\sigma_{x}$ dominates $\mathbb{1}$, in which case $\phi_{c} \leq \phi_{A} \leq$ $2 \arctan \left(\Lambda_{\alpha} /\left|\cos \theta_{B}\right|\right)$, the solution is obtained for the situation in which Alice plays $\mathbf{U}$ and Bob plays $\sigma_{x}$, which leads Alice to receive a payment equal to that shown in Eq. (62).

(iii) In the case where Alice's quantum strategy $\mathbf{U}$ dominates only $\sigma_{x}$, defined by the interval $2 \arctan \left(\max \left\{1 / \Lambda_{\alpha}, \Lambda_{\alpha} /\left|\cos \theta_{B}\right|\right\}\right) \leq \phi_{A}<\pi$, the game solution depends on the dominance regime between Bob's strategies:

(a) When Bob's classical strategy $\mathbb{1}$ dominates $\sigma_{x}$, that is,

$$
\pi(\mathbb{1}, \mathbf{x}) \geq \pi(\mathbb{1}, \mathbb{1}) \text { and } \pi(\mathbf{U}, \mathbf{x}) \geq \pi(\mathbf{U}, \mathbb{1})
$$

which occurs whenever $2 \arctan \left(\max \left\{1 / \Lambda_{\alpha}, \Lambda_{\alpha} /\left|\cos \theta_{B}\right|\right\}\right) \leq \phi_{A} \leq \phi_{c}$, one should discard $\sigma_{x}$ in our analysis. Thus, it is verified that the Alice's quantum strategy $\mathbf{U}$ starts to dominate $\mathbb{1}$, because in this reduced space of strategies, we have that $\pi(\mathbf{U}, \mathbb{1}) \geq \pi(\mathbb{1}, \mathbb{1})$, according to (36a). Therefore, the game solution is obtained with Alice by playing $\mathbf{U}$ and Bob $\mathbb{1}$, providing a payment to Alice according to Eq. (63).

(b) When there is no dominance between Bob's classical strategies, which occurs in the interval $\phi_{c}<2 \arctan \left(\max \left\{1 / \Lambda_{\alpha}, \Lambda_{\alpha} /\left|\cos \theta_{B}\right|\right\}\right) \leq \phi_{A}$, the game solution becomes identical to that of item (b) of (iii) for the regime in which $\left|\alpha_{0}\right| \geq\left|\alpha_{1}\right|$, discussed above. Therefore, we must adjust the probabilities according to (55), which leads Alice to receive a payment given by (56).

(iv) When there is no dominance between any of Bob's and Alice's strategies, defined by the interval $2 \arctan \left(\Lambda_{\alpha} /\left|\cos \theta_{B}\right|\right)<\phi_{A}<2 \arctan \left(1 / \Lambda_{\alpha}\right)$, we recover the same situation occurred in (iv) in the regime where $\left|\alpha_{0}\right| \geq\left|\alpha_{1}\right|$, analyzed above. Therefore, the solution is separated into two cases:

(a) If $2 \arctan \left(\Lambda_{\alpha} /\left|\cos \theta_{B}\right|\right)<\phi_{A} \leq \phi_{c}$ we must make the adjustment in (59), which leads Alice to receive a payment defined by Eq. (51).

(b) If $\phi_{c} \leq \phi_{A}<2 \arctan \left(1 / \Lambda_{\alpha}\right)$ we must make the adjustment defined in Eq. (60), which leads Alice to receive a payment given by Eq. (56).

Although the phase relation between the initial superposition states are very important, in this work we will disregard these effects, making them zero. The effects of this phase will be analyzed in a future work. 


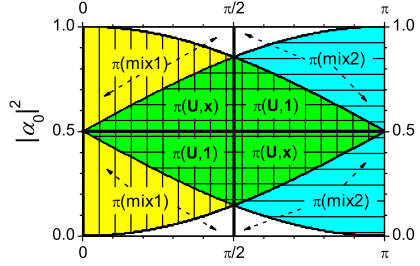

(a) $\theta_{A}=\theta_{B}=\pi$

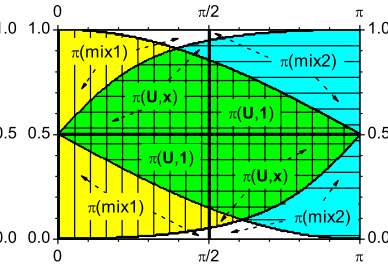

(b) $\theta_{A}=\pi ; \theta_{B}=2 \pi / 3$

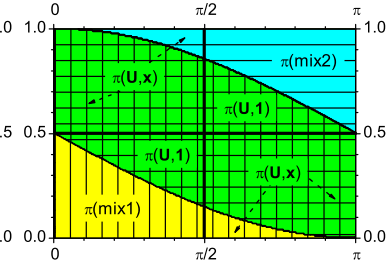

(c) $\theta_{A}=\pi ; \theta_{B}=\pi / 2$

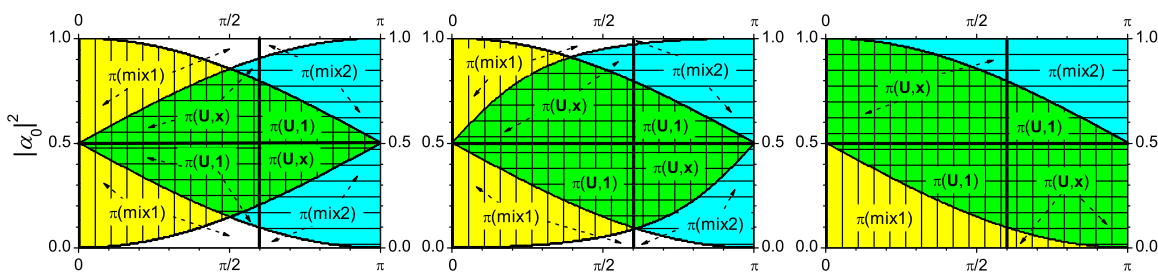

(d) $\theta_{A}=2 \pi / 3 ; \theta_{B}=\pi$

(e) $\theta_{A}=\theta_{B}=2 \pi / 3$

(f) $\theta_{A}=2 \pi / 3 ; \theta_{B}=\pi / 2$

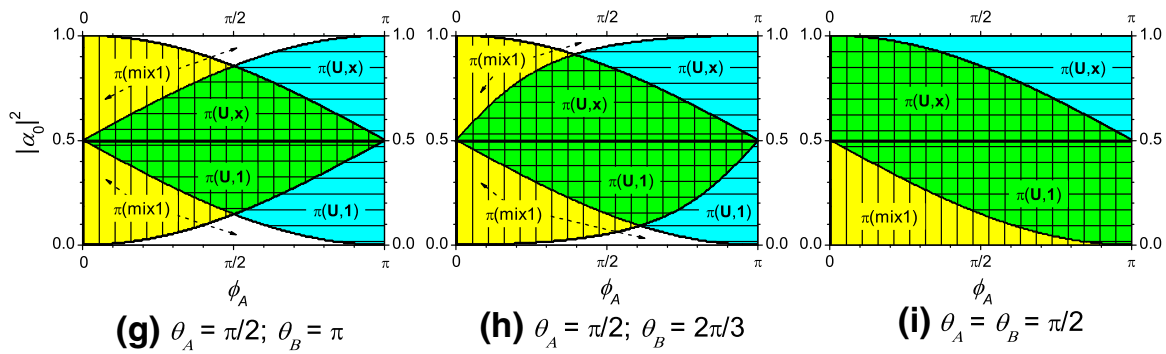

Fig. 1 Regions of dominance for Alice's quantum strategy $\mathbf{U}$ when we vary the initial game state parameter $\left|\alpha_{0}\right|^{2}$ and the parameter $\phi_{A}$, associated with the Alice's quantum strategy $\mathbf{U}$. The yellow region hatched with vertical lines represents the domain of $\mathbf{U}$ over the classical strategy $\mathbb{1}$; the cyan region hatched with horizontal lines represents the domain of $\mathbf{U}$ over the classical strategy $\sigma_{x}$; and the green region hatched with horizontal and vertical lines represents the mutual domain of $\mathbf{U}$ over the classical strategies $\mathbb{1}$ and $\sigma_{x}$. The thick vertical line represents the critical value $\phi=\phi_{c}$, while the thick horizontal line represents the critical value for $\left|\alpha_{0}\right|^{2}=0.5$. Alice's payments are indicated in each of the sectors delimited by dominance regions and critical lines. In this figure, we setting the parameters: $\mathbf{a} \theta_{A}=\theta_{B}=\pi ; \mathbf{b} \theta_{A}=\pi$ and $\theta_{B}=2 \pi / 3$; $\theta_{A}=\pi$ and $\theta_{B}=\pi / 2 ; \mathbf{d} \theta_{A}=2 \pi / 3$ and $\theta_{B}=\pi ; \mathbf{e} \theta_{A}=\theta_{B}=2 \pi / 3 ; \mathbf{f} \theta_{A}=2 \pi / 3$ and $\theta_{B}=\pi / 2 ; \mathbf{g}$ $\theta_{A}=\pi / 2$ and $\theta_{B}=\pi ; \mathbf{h} \theta_{A}=\pi / 2$ and $\theta_{B}=2 \pi / 3 ; \mathbf{i} \theta_{A}=\theta_{B}=\pi / 2$ (Color figure online)

For a better understanding of the emergence of transitions between optimal strategies that we'll see in the next section, it is imperative that we visualize where each game solution is located in the space formed by the $\left|\alpha_{0}\right|^{2}$ and $\phi_{A}$ parameters. For this purpose, in Fig. 1 we show the dominance regions for Alice's quantum strategy $\mathbf{U}$ in a space defined by $\left|\alpha_{0}\right|^{2}$ and $\phi_{A}$. We also put the game solution for each of the sectors delimited by dominance regions and critical lines, defined by $\phi_{A}=\phi_{c}$ (vertical line) and $\left|\alpha_{0}\right|^{2}=0.5$ (horizontal line). Imagining these graphs as elements of a $3 \times 3$ matrix, from left to right, we set $\theta_{B}$ as $\theta_{B}=\pi$ (column 1), $\theta_{B}=2 \pi / 3$ (column 2) and $\theta_{B}=\pi / 2$ (column 3), while from top to bottom, we set the value of $\theta_{A}$ as $\theta_{A}=\pi$ (line 1), $\theta_{A}=2 \pi / 3$ (line 2) and $\theta_{A}=\pi / 2$ (line 3). From Eq. (39), when $\left|\alpha_{0}\right|^{2}<0.5$, we note that the $\mathbf{U}$ dominance over classical strategy $\mathbb{1}$ is widened (superior limit) when $\theta_{B}$ 
decreases, whereas from Eq. (42), when $\left|\alpha_{0}\right|^{2}>0.5$, we observe that the dominance of $\mathbf{U}$ over the classical strategy $\sigma_{x}$ is widened (inferior limit) when $\theta_{B}$ decreases. As a consequence, we observe that the region of mutual dominance increases along of line that unify the points $\left(\left|\alpha_{0}\right|^{2}=1, \phi_{A}=0\right)$ and $\left(\left|\alpha_{0}\right|^{2}=0, \phi_{A}=\pi\right)$. It is also noted that the decrease in $\theta_{A}$ does not change the dominance regions, although it alters the critical points $\phi_{c}$ and consequently the solutions in each of the sectors. Note that as $\phi_{c}$ moves to the right, the game solutions to the left of it also extend to the right.

Now we are ready to analyze the payment functions when we vary the parameters of the adiabatic evolution $\theta_{A}$ and $\theta_{B}$, the parameter of the Alice's quantum strategy $\phi_{A}$, and the parameter of the initial state $\left|\alpha_{0}\right|^{2}$.

\section{Transitions between optimal strategies}

Since the functional form of Alice's payment function $\Pi_{A}$ depends on the interval at which $\phi_{A}$ is adjusted, we may be suspected that there are discontinuities in this function or in its derivatives. In fact, as we shall see below, the payment function, although continuous, presents discontinuous derivatives that characterize or signalize a transition between optimal strategies in a very similar way with phase transitions in thermodynamics.

In Fig. 2, we plot Alice's payment $\Pi_{A}$ as a function of $\left|\alpha_{0}\right|^{2}$ and considering different fixed values of $\phi_{A}$ and $\theta_{B}$. In this figure, we consider that Alice receives from Bob twice more than she pays to him, i.e., $x_{0}=2 x_{1}$, and we choose: (a) $\theta_{A}=\pi$, (b) $\theta_{A}=2 \pi / 3$ and (c) $\theta_{A}=\pi / 2$. In Fig. 2 a we note five important points:

(i) For any of the curves, we note that the maximum value of $\Pi_{A}$ occurs when $\left|\alpha_{0}\right|^{2}=0.5$ and the minimum value occurs both when $\left|\alpha_{0}\right|^{2}=0$ and 1 .

(ii) The curve associated with the best gains occur when we set Alice's quantum strategy parameter for $\phi_{A}=\pi / 2$ and the worst gain for $\phi_{A}=0$ or $\pi$.

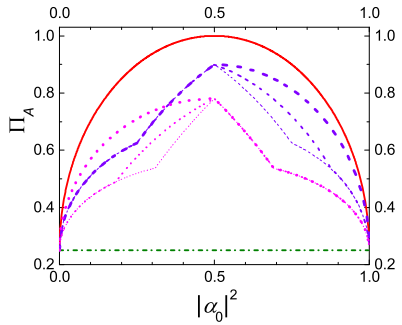

(a) $\theta_{A}=\pi$

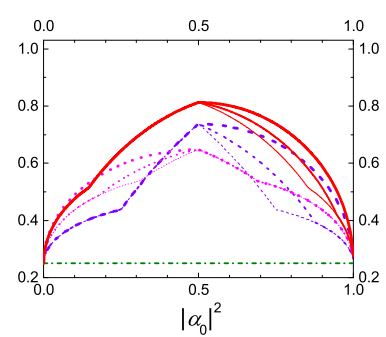

(b) $\theta_{A}=2 \pi / 3$

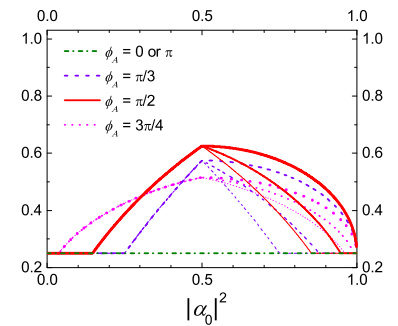

(c) $\theta_{A}=\pi / 2$

Fig. 2 The Alice's payment $\Pi_{A}$ as a function of the initial state parameter $\left|\alpha_{0}\right|^{2}$. Fixing $x_{0}=1$ and $x_{1}=0.5$, the curves represent the parameter set defined by: $\phi_{A}=0$ or $\pi$ (green dash-dot line); $\phi_{A}=\pi / 3$ with $\theta_{B}=\pi / 2$ (purple thick dashed line), $\theta_{B}=2 \pi / 3$ (purple dashed line) and $\theta_{B}=\pi$ (purple thin dashed line); $\phi_{A}=\pi / 2$ (red solid line); and finally $\phi_{A}=3 \pi / 4$ with $\theta_{B}=\pi / 2$ (pink thick dotted line), $\theta_{B}=2 \pi / 3$ (pink dotted line) and $\theta_{B}=\pi$ (pink thin dotted line). In a-c we choose, respectively, the parameters $\theta_{A}=\pi, 2 \pi / 3$ and $\pi / 2$ (Color figure online) 
(iii) For $\phi_{A}=\pi / 3$, we observed that as $\theta_{B}$ decreases the Alice's gains increase only when $\left|\alpha_{0}\right|^{2}>0.5$, while for $\phi_{A}=3 \pi / 4$, as $\theta_{B}$ decreases the Alice's gains increase only when $\left|\alpha_{0}\right|^{2}<0.5$.

(iv) We observed that the curves associated with $\phi_{A}=\pi / 3$ (purple dashed line) and $\phi_{A}=3 \pi / 4$ (pink dotted line) can cross each other for $\theta_{B}=\pi / 2$.

(v) Except for cases $\phi_{A}=0, \pi / 2$, and $\pi$, we see the emergence of discontinuities in the derivatives of $\Pi_{A}$ in some points of the curves.

For the first point (i) we would like to remember that when $\left|\alpha_{0}\right|^{2}=0.5$ we have only the regime of mutual dominance and for $\left|\alpha_{0}\right|^{2}=0$ or 1 we have only the regime of absence of dominance according to Alice's point of view (see Fig. 1). The same occurs in Fig. 2b. In Fig. 2c, for any curve of the graph, we see the emergence of a range of $\left|\alpha_{0}\right|^{2}$ in which the $\Pi_{A}$ value is also minimal. In these intervals, the game solution comes from the solution $\pi$ (mix 1 ) (see Fig. $1 \mathrm{~g}-\mathrm{i}$ ), which for $\theta_{A}=\pi / 2$ results in a constant minimum payment $\left(x_{0}-x_{1}\right) / 2=0.25$. With respect to the second point (ii), it is easy to understand that $\phi_{A}=0$ or $\pi$ leads to the worst gain because in this situation the quantum strategy becomes classical. Also when $\left|\alpha_{0}\right|^{2}=0.5$ we expected that $\phi_{A}=\pi / 2$ leads to the best strategy for Alice, because this was the (ideal) case we discussed at the beginning of the previous section. For other values of $\left|\alpha_{0}\right|^{2}$, this conclusion is no longer true if $\theta_{A} \neq \pi$, as is evident by the pink curves intercepting the red curves in Fig. 2b, c. In the third point (iii), we observed an apparent non-intuitive behavior, since one could expect that the smaller the efficiency in the application of Bob's strategy, the greater the Alice's payments along the whole spectrum of variation of $\left|\alpha_{0}\right|^{2}$. But this reasoning is erroneous, since while Bob's strategy $\sigma_{x}$ depends on machine efficiency, the strategy $\mathbb{1}$ is immune. Therefore, the part of the curve that undergoes changes with $\theta_{B}$ originates from the solution $\pi$ (U, $\mathbf{x}$ ) (see Eq. (52)), while the other parts comes from other solutions that do not depend on $\theta_{B}$ (see Fig. 1g, h) or (i) for the parameter sets of each curve in Fig. 2c). The fourth point (iv) is very important in situations where there are imperfections in the implementation of each strategies. The fact that the curves cross each other means that there are different optimal $\phi_{A}$ settings for different $\left|\alpha_{0}\right|^{2}$ values. Therefore, in the presence of these machines imperfections we cannot think that the closer to the ideal strategy $\phi_{A}=\pi / 2$ the better (see pink curves in Fig. 2b, c). For the last point (v), excluding the situation where the quantum strategy becomes classical $\left(\phi_{A}=0\right.$ and $\left.\pi\right)$, we note that these discontinuities are associated with situations in which the transitions occur between optimal strategies as we can see from Fig. 1 for each parameter set of each curves in Fig. 2. The fact that there are no discontinuities for $\phi_{A}=\pi / 2$ is because both $\phi_{A}$ and the critical parameter $\phi_{c}$ have the same value $\phi_{A}=\phi_{c}=\pi / 2$ when $\theta_{A}=\pi$ (see Fig. 1a, d, g). Therefore, when we varies $\left|\alpha_{0}\right|^{2}$, we are remaining on the border that separates different strategies and we do not crossing them. On the other hand, we see from Fig. 2b, $c$ that the strategy transitions arise as a consequence of $\phi_{A}=\pi / 2 \neq \phi_{c}$ for $\theta_{A} \neq \pi$, as we can see from Fig. $1 \mathrm{~d}-\mathrm{i}$.

In Fig. 3, we plot Alice's payment $\Pi_{A}$ as a function of $\phi_{A}$ and considering different fixed values of $\left|\alpha_{0}\right|^{2}$ and $\theta_{B}$. In this plot we consider again $x_{0}=2 x_{1}$ and we choose: (a) $\theta_{A}=\pi$, (b) $\theta_{A}=2 \pi / 3$ and (c) $\theta_{A}=\pi / 2$. In Fig. 3a, we observe five similar details to Fig. 2a: 


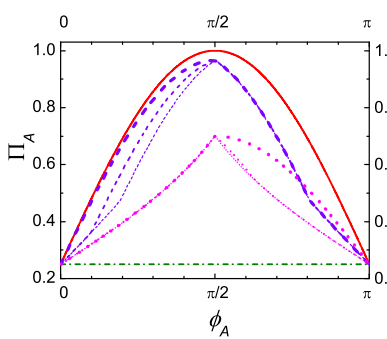

(a) $\theta_{A}=\pi$

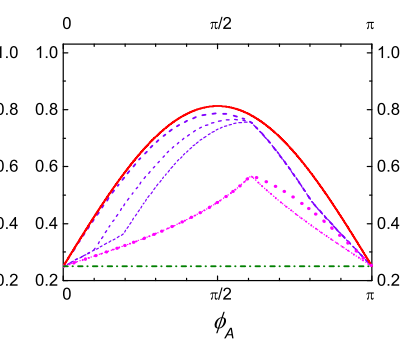

(b) $\theta_{A}=2 \pi / 3$

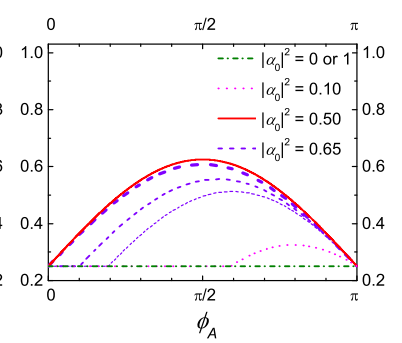

(c) $\theta_{A}=\pi / 2$

Fig. 3 The Alice's payment $\Pi_{A}$ as a function of the Alice's quantum strategy parameter $\phi_{A}$. Fixing $\theta_{A}=\pi$, $x_{0}=1$, and $x_{1}=0.5$, the curves represent the parameter set defined by: $\left|\alpha_{0}\right|^{2}=0$ or 1 (green dash-dot line); $\left|\alpha_{0}\right|^{2}=0.1$ with $\theta_{B}=\pi / 2$ (pink thick dotted line), $\theta_{B}=2 \pi / 3$ (pink dotted line) and $\theta_{B}=\pi$ (pink thin dotted line); $\left|\alpha_{0}\right|^{2}=0.5$ (red solid line); and finally $\left|\alpha_{0}\right|^{2}=0.65$ with $\theta_{B}=\pi / 2$ (purple thick dashed line), $\theta_{B}=2 \pi / 3$ (purple dashed line) and $\theta_{B}=\pi$ (purple thin dashed line). In a-c we choose, respectively, the parameters $\theta_{A}=\pi, 2 \pi / 3$ and $\pi / 2$ (Color figure online)

(i) For any of the curves, we note that the maximum value of $\Pi_{A}$ occurs when $\phi_{A}=\pi / 2$ and the minimum value occurs both when $\phi_{A}=0$ and $\pi$.

(ii) The curve associated with the best gains occur when the initial game state parameter is $\left|\alpha_{0}\right|^{2}=0.5$ and the worst gain when $\left|\alpha_{0}\right|^{2}=0$ or 1 .

(iii) For $\left|\alpha_{0}\right|^{2}=0.10$, we observed that as $\theta_{B}$ decreases the Alice's gains increase only when $\phi_{A}>\pi / 2$, while for $\left|\alpha_{0}\right|^{2}=0.65$, as $\theta_{B}$ decreases the Alice's gains increase only when $\phi_{A}<\pi / 2$.

(iv) We observed that the curves associated with $\left|\alpha_{0}\right|^{2}=0.65$ (purple dashed line) and $\left|\alpha_{0}\right|^{2}=0.10$ (pink dotted line) can cross each other for $\theta_{B}=\pi / 2$.

(v) Except for cases $\left|\alpha_{0}\right|^{2}=0,0.5$ and 1, we see the emergence of discontinuities in the derivatives of $\Pi_{A}$ in some points of the curves.

The point (i) can be understood by the fact that both $\phi_{A}=0$ and $\pi$ make $\mathbf{U}$ becomes a classical strategy. For $\theta_{A}=\pi$, we have $\phi_{A}=\phi_{c}=\pi / 2$, so that this is a maximum point for any curve. For other $\theta_{A}$ values, this conclusion is no longer valid, as shown by pink dotted lines in Fig. 3b, c, for $\left|\alpha_{0}\right|^{2} \neq 0.5$. The second point (ii) is justified because for $\left|\alpha_{0}\right|^{2}=0$ and 1 there is a total absence of dominance and for $\left|\alpha_{0}\right|^{2}=0.5$ there is mutual dominance in all $\phi_{A}$ spectrum for any $\theta_{A}$ and $\theta_{B}$, as we can see in Fig. 1 . The comments about points (iii) and (iv) are identical to that of Fig. 2 that we performed above. For the last point (v), we must remember that for $\left|\alpha_{0}\right|^{2}=0$ and 1 we have total absence of mutual dominance, such that we can discard the quantum strategy, that is, in these conditions the game resembles the classic game. In any other situation we have a quantum game where there are transitions between optimal strategies. The fact that transitions do not appear for $\left|\alpha_{0}\right|^{2}=0.5$ is because this value is a critical point (see Fig. 1), so that we fall into the same situation that occurred in point (v) of the previous figure. In general, if we walk along a horizontal or vertical line, we can see that there will be at most 3 singularities, whereas walking along the critical lines will not exist at all.

The points (i) and (ii) can be better understood with Fig. 4, where we show the level curves for $\Pi_{A}$ function in the space defined by $\phi_{A}$ and $\left|\alpha_{0}\right|^{2}$, using the same parameters 


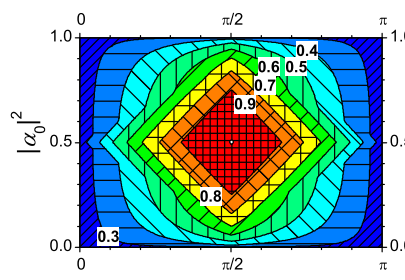

(a) $\theta_{A}=\theta_{B}=\pi$

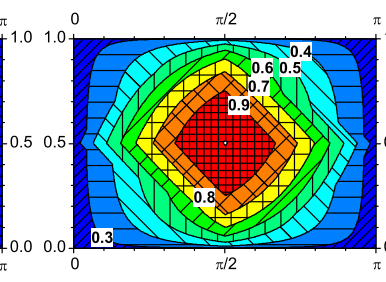

(b) $\theta_{A}=\pi ; \quad \theta_{B}=2 \pi / 3$

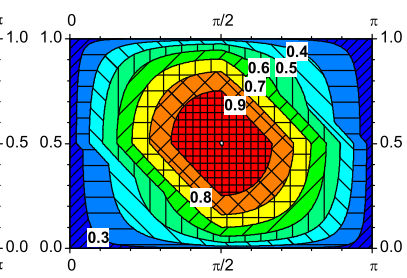

(c) $\theta_{A}=\pi ; \quad \theta_{B}=\pi / 2$

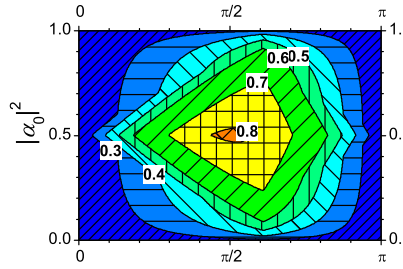

(d) $\theta_{A}=2 \pi / 3 ; \theta_{B}=\pi$

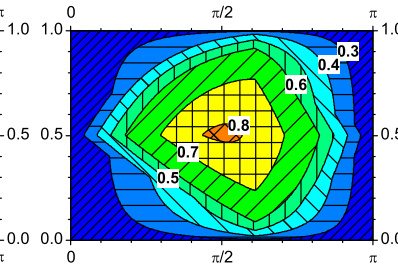

(e) $\theta_{A}=\theta_{B}=2 \pi / 3$

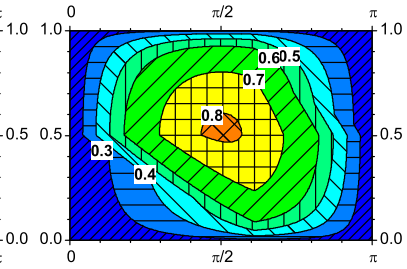

(f) $\theta_{A}=2 \pi / 3 ; \theta_{B}=\pi / 2$

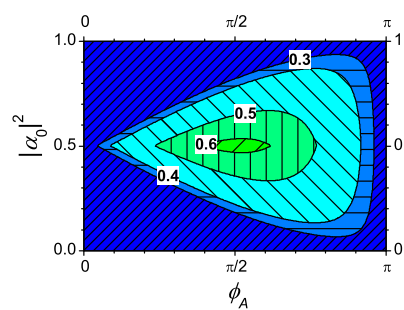

(g) $\theta_{A}=\pi / 2 ; \theta_{B}=\pi$

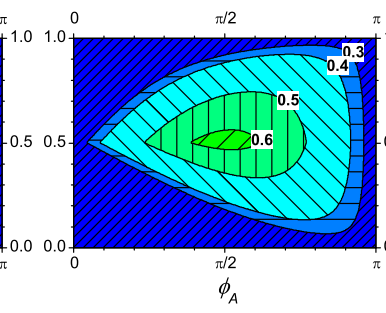

(h) $\theta_{A}=\pi / 2 ; \theta_{B}=2 \pi / 3$

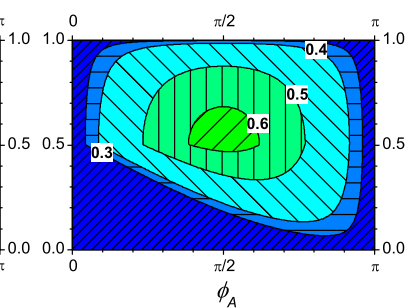

(i) $\theta_{A}=\theta_{B}=\pi / 2$

Fig. 4 The level curves of Alice's payment function $\Pi_{A}$ in a region defined by the parameters associated with the initial state, $\left|\alpha_{0}\right|^{2}$, and the Alice's quantum strategy, $\phi_{A}$. We setting $x_{0}=1$ and $x_{1}=0.5$ and: $\mathbf{a} \theta_{A}=\theta_{B}=\pi ; \mathbf{b} \theta_{A}=\pi$ and $\theta_{B}=2 \pi / 3 ; \mathbf{c} \theta_{A}=\pi$ and $\theta_{B}=\pi / 2 ; \mathbf{d} \theta_{A}=2 \pi / 3$ and $\theta_{B}=\pi ; \mathbf{e}$ $\theta_{A}=\theta_{B}=2 \pi / 3 ; \mathbf{f} \theta_{A}=2 \pi / 3$ and $\theta_{B}=\pi / 2 ; \mathbf{g} \theta_{A}=\pi / 2$ and $\theta_{B}=\pi ; \mathbf{h} \theta_{A}=\pi / 2$ and $\theta_{B}=2 \pi / 3 ; \mathbf{i}$ $\theta_{A}=\theta_{B}=\pi / 2$

configuration for $\theta_{A}$ and $\theta_{B}$ in Fig. 1, besides the adjustment $x_{0}=2 x_{1}=1$. Note that the highest gain region is concentrated where there is mutual dominance of strategy $\mathbf{U}$. We also see that as $\theta_{B}$ decreases the region of higher gains increases as a consequence of the amplification of the mutual dominance region. When $\theta_{A}$ decreases, we see that Alice's gains decrease as a consequence of decreased efficiency in implementing $\mathbf{U}$ strategy. Note that the graphs of the first line $\left(\theta_{A}=\pi\right)$ have reflection symmetry on the axes $\phi_{c}=\pi / 2$ and $\left|\alpha_{0}\right|^{2}=0.5$, which are the critical points. While the critical points for $\left|\alpha_{0}\right|^{2}=0.5$ do not change in any situation (hence the maximum payoff always occurs at $\left|\alpha_{0}\right|^{2}=0.5$ ), the critical points for $\phi_{c}$ move to the right as $\theta_{A}$ decreases. As a consequence, the maximum value, for some $\left|\alpha_{0}\right|^{2}$ values farther from $\left|\alpha_{0}\right|^{2}=0.5$, to move to the right.

Finally, we would like to point out that the transitions between the players' optimal strategies (TBOS) in $\Pi_{A}$, which resemble the phase transitions in thermodynamics, differ from those mentioned in the literature involving games with 2 qubits in the presence of entanglement $[47,54]$ and without entanglement [55], but with interacting 
qubits. In this paper, the TBOS are not consequences of entanglement, but rather essentially of the superposition of the states $|0\rangle$ and $|1\rangle$.

Using the phase transition formalism in thermodynamics, we can verify that the order parameter in a TBOS is a function of $\Pi_{A}$, having as main critical points $\left|\alpha_{0}\right|^{2}=$ 0.5 and $\phi_{A}=\phi_{c}$, besides the critical points associated with the lines that delimit the domain regions between the strategies, as shown in Fig. 1. Note that in the transition between the strategies $\pi(\mathbf{U}, \mathbf{x}) \rightarrow \pi(\mathbf{U}, \mathbb{1})$, that in the vicinity of the critical point $\phi_{A}=\phi_{c}$, taking $\theta_{A}=\pi$, we have

$$
\begin{aligned}
& \pi(\mathbf{U}, \mathbf{x})-\pi(\mathbf{U}, \mathbb{1}) \\
& \quad=-\left(x_{0}+x_{1}\right)\left(\left|\cos \theta_{B}\right|+1\right)\left(\left|\alpha_{0}\right|^{2}-\frac{1}{2}\right)\left[1-2 \sin ^{2}\left(\frac{\phi_{A}}{2}\right)\right] \\
& \quad \simeq\left(x_{0}+x_{1}\right)\left(\left|\cos \theta_{B}\right|+1\right)\left(\left|\alpha_{0}\right|^{2}-\frac{1}{2}\right)\left(\phi_{A}-\phi_{c}\right),
\end{aligned}
$$

which shows that the critical exponent becomes 1. The same critical exponent is obtained for $\left|\alpha_{0}\right|^{2} \rightarrow 0.5$. In addition, from Eq. (65), we see that the functions $\pi(\mathbf{U}, \mathbf{x})$ and $\pi(\mathbf{U}, \mathbb{1})$ assume equal values at the critical points $\left|\alpha_{0}\right|^{2}=0.5$ and $\phi_{A}=\phi_{c}$, while their derivatives diverge at this point by a constant value.

\section{Summary and conclusions}

In this work, using controlled adiabatic evolutions, we show how to implement classical and quantum strategies in a two-person, zero-sum "spin flip game", "even or odd" and "heads or tails" game. Through the formalism of controlled adiabatic evolutions, it is possible to schematize the game in a circuit model that is independent of the physical platform used and, therefore, our results are independent of this physical system. Another interesting factor that arises when introducing this adiabatic model is the appearance of a probabilistic parameter $\theta$ that we can associate with the efficiency of the machine that implements the strategies in the game. When this parameter $\theta=\pi$, in which case the machine implements the strategies with maximum efficiency, we recover the usual situation that occurs in "heads or tails" games seen in the literature. In spite of this new ingredient, this work goes further and analyzes game situations in which the initial state is a superposition state of a single qubit, of the form $\alpha_{0}\left|0_{c}\right\rangle+$ $\alpha_{1}\left|1_{c}\right\rangle$, where we leave the coefficients $\alpha_{0,1}$ varies from 0 to 1 , such that $\left|\alpha_{0}\right|^{2}+\left|\alpha_{1}\right|^{2}=$ 1. In addition, we consider situations in which each player receives from each other, different payments, say $x_{0}$ for Alice and $x_{1}$ for Bob. Below we summarize the main points of this work.

(i) We initially consider the situation in which the game starts with the state $\left|0_{c}\right\rangle$ or $\left|1_{c}\right\rangle$ and players can only use classical strategies $\left\{\mathbb{1}, \sigma_{x}\right\}$. We note that the adjustment of the optimal strategies for both players requires the prior knowledge of $\theta$ and, as a consequence, the payments become independent of this parameter, being $\left(x_{0}-x_{1}\right) / 2$ for Alice and $\left(x_{1}-x_{0}\right) / 2$ for Bob. When $x_{0}>x_{1}\left(x_{0}<x_{1}\right)$ Alice has (dis)advantages of classical origin, that is, (dis)advantages that would 
occur in a purely classical game. When $x_{0}=x_{1}$, both players have null payment, in which case we say that the game is fair.

(ii) If we allow in the previous situation (i) one or both players can add a quantum strategy $\mathbf{U}$, it turns out that the adjustment to obtain the optimal strategy can be done in different ways, including neglecting the quantum strategy. For this reason, the payment of both players does not change with respect to case (i) and therefore there are no advantages for any player.

(iii) Differently from cases (i) and (ii), we now proceed to analyze the game from an initial superposition state $\alpha_{0}\left|0_{c}\right\rangle+\alpha_{1}\left|1_{c}\right\rangle$. For this situation, we consider that Alice has the strategies $\left\{\mathbb{1}, \sigma_{x}, \mathbf{U}\right\}$ and Bob only the classical strategies $\left\{\mathbb{1}, \sigma_{x}\right\}$. In this new scenario, we analyze Alice's gains for different games, each of them varying the parameters $\left|\alpha_{0}\right|^{2}$, associated with the initial game state, and $\phi$, associated with unitary rotation $\mathbf{U}$. In the situation where $\theta_{A}=\pi$ (efficiency of $100 \%$ of the Alice's machine), we note that the Alice's maximum gains occur when $\left|\alpha_{0}\right|^{2}=1 / 2$ and $\phi=\pi / 2$. However, when $\theta_{A} \neq \pi$, and $\left|\alpha_{0}\right|^{2} \neq 0.5$, we note that the best fit happens for $\phi \neq \pi / 2$.

(iv) Finally, we note that by varying the parameters $\left|\alpha_{0}\right|^{2}$ and $\phi$, optimal strategies can undergo transitions similarly to phase transitions in thermodynamics. As a consequence of these transitions, the payments of the quantum player, although they vary continuously, undergo an abrupt change in its derivative, which causes a greater increase or decrease in the gains when we vary $\left|\alpha_{0}\right|^{2}$ and $\phi$. It is important to note that these transitions occur for a game with a single qubit, contrary to what is mentioned in the literature when two-qubit games are analyzed, and therefore entanglement plays an important role.

To conclude this work, we would like to point out that (iii), highlighted above, shows that the best strategies of a quantum game depends on the machine efficiency that implements them. For this reason, we believe that adiabatic formalism may be an additional tool for analyzing any real game in addition to providing new horizons (e.g., use of alternative adiabatic degenerate Hamiltonians in quantum annealers $[30,56]$ or digitalized quantum adiabatic computation [29]) for the implementation of strategies in a game.

Finally, according to Fig. 1, we see that depending on the configuration of the game parameters $\left(\phi_{A}\right.$, associated with the quantum strategy, and $\left|\alpha_{0}\right|^{2}$, associated with the initial game state) it is indispensable to consider the whole strategies set for both players, since the optimal solution of the game is obtained by mixed strategies and no longer by pure strategies, such as most of the games analysis done in the literature.

Acknowledgements A.C.S. acknowledges financial support from the Brazilian agencies CNPq and the Brazilian National Institute of Science and Technology for Quantum Information (INCT-IQ), and M. A. P. would like to thank God for the opportunity to do this work.

\section{References}

1. dos Santos, T.B., Peixoto, P.E.: Agonistic interactions in the dragonfly Micrathyria ungulata: does male fighting investment come from an innate ability or an indomitable will? Behav. Ecol. Sociobiol. 71, 104 (2017) 
2. Smith, J.M.: Evolution and the Theory of Games, vol. viii. Cambridge University Press, Cambridge (1982)

3. Morris, A., MacGlashan, J., Littman, M.L., Cushman, F.: Evolution of flexibility and rigidity in retaliatory punishment. PNAS 114, 10396-10401 (2017)

4. Rilling, J.K., Gutman, D.A., Zeh, T.R.: A neural basis for social cooperation. Neuron 35, 395-405 (2002)

5. Rilling, J.K., et al.: Neural correlates of social cooperation and non-cooperation as a function of psychopathy. Biol. Psychiat. 61, 1260-1271 (2007)

6. Kar, R.B.: The evolutionary game-theoretic foundations of law. Law Soc. Inq. 42, 38-48 (2017)

7. Fischer, I., Suleiman, R.: Election frequency and the emergence of cooperation in a simulated intergroup conflict. J. Confl. Resolut. 41, 483-508 (1997)

8. Streich, P., Levy, J.S.: Time horizons, discounting, and intertemporal choice. J. Confl. Resolut. 51, 199-226 (2007)

9. Chiu, C.-P., Lai, S.-K.: A comparison of regimes of policies: lessons from the two-person iterated prisoner's dilemma game. Environ. Plan. B 35, 794-809 (2008)

10. Vassiliades, V., Cleanthous, A., Christodoulou, C.: Multiagent reinforcement learning: spiking and nonspiking agents in the iterated prisioner's dilemma. IEEE Trans. Neural Netw. 22, 639-653 (2011)

11. Plous, S.: The nuclear arms race: prisoner's dilemma or perceptual dilemma? J. Peace Res. 30, 163-179 (1993)

12. Pajunen, K.: Living in agreement with a contract: the management of moral and viable firm-stakeholder relationships. J. Bus. Ethics 68, 243-258 (2006)

13. Clempner, J.B.: A game theory model for manipulation based on machiavellianism: moral and ethical behavior. JASSS 20, 12 (2017)

14. Chiong, R., Wong, D.M.L., Jankovic, L.: Agent-based economic modelling with iterated prisioner's dilemma. ICOCI 2006, 1-6 (2006)

15. Chiong, R.: Applying genetic algorithms to economy market using iterated prisoner's dilemma. Appl. Comput. 1-2, 733-737 (2007)

16. Baxa, J., Brozova, H., Kvasnicka, R.: Cooperative behavior and economic growth. Math. Methods Econ. 2009, 13-18 (2009)

17. Perez-Castrillo, D., Sotomayor, M.: On the manipulability of competitive equilibrium rules in manyto-many buyer-seller markets. Int. J. Game Theory 46, 1137-1161 (2017)

18. Krach, S., et al.: Can machines think? Interaction and perspective taking with robots investigated via fMRI. Plos One 3(e2597), 1-11 (2008)

19. Asher, N., Paul, S., Venant, A.: Message exchange games in strategic contexts. J Philos Logic 46, 355-404 (2017)

20. Shinar, J.: Differential games and artificial intelligence-A new approach for solving complex dynamic conflicts. Lecture Notes in Control and Information Sciences 156, 100-110 (1991)

21. Basilico, N., Gatti, N., Amigoni, F.: Patrolling security games: definition and algorithms for solving large instances with single patroller and single intruder. Artif. Intell. 184, 78-123 (2012)

22. Kargarian, A., et al.: Artificial intelligence-based loss allocation algorithm in open access environment. J. Energy Eng. 140, 04013021 (2014)

23. Pease, A., et al.: Lakatos-style collaborative mathematics through dialectical, structured and abstract argumentation. Artif. Intell. 246, 181-219 (2017)

24. Lee, C.F., Johnson, N.: Parrondo games and quantum algorithms, pp. 1-7 (2002). arxiv:quant-ph/0203043v1

25. Doscher, C., Keyl, M.: An introduction to quantum coin tossing. Fluct Noise Lett. 2, R125-R137 (2002)

26. Gogonea, V., Merz, K.M.: Fully quantum mechanical description of proteins in solution. Combining linear scaling quantum mechanical methodologies with the Poisson-Boltzmann equation. J. Phys. Chem. A 103, 5171-5188 (1999)

27. Berkley, A.J., et al.: A scalable readout system for a superconducting adiabatic quantum optimization system. Supercond. Sci. Technol. 23, 105014 (2010)

28. Johnson, M.W., et al.: A scalable control system for a superconducting adiabatic quantum optimization processor. ibid 23, 065004 (2010)

29. Barends, R., et al.: Digitized adiabatic quantum computing with a superconducting circuit. Nature 534, 222-226 (2016) 
30. Boixo, S., et al.: Evidence for quantum annealing with more than one hundred qubits. Nat. Phys. 10, 218-224 (2014)

31. Friedenauer, A., et al.: Simulating a quantum magnet with trapped ions. Nat. Phys. 4, 757-761 (2008)

32. Kim, K., et al.: Quantum simulation of frustrated Ising spins with trapped ions. Nature 465, 590-593 (2010)

33. Mitra, A., et al.: Experimental implementation of local adiabatic evolution algorithms by an NMR quantum information processor. J. Magn. Reson. 177, 285-298 (2005)

34. Steffen, M., et al.: Experimental implementation of an adiabatic quantum optimization algorithm. Phys. Rev. Lett. 90, 067903 (2003)

35. Peng, X., et al.: Quantum adiabatic algorithm for factorization and its experimental implementation. Phys. Rev. Lett. 101, 220405 (2008)

36. $\mathrm{Xu}, \mathrm{K}$., et al.: Experimental adiabatic quantum factorization under ambient conditions based on a solid-state single spin system. Phys. Rev. Lett. 118, 130504 (2017)

37. Klein, J., Beil, F., Halfmann, T.: Robust population transfer by stimulated raman adiabatic passage in a $\mathrm{Pr}_{3}+: \mathrm{Y}_{2} \mathrm{SiO}_{5}$ crystal. Phys. Rev. Lett. 99, 113003 (2007)

38. Born, M., Fock, V.: Beweis des Adiabatensatzes. Z. Phys. A Hadron Nucl. 51, 165-180 (1928)

39. Kato, T.: On the Adiabatic theorem of quantum mechanics. J. Phys. Soc. Jpn. 5, 435-439 (1950)

40. Messiah, A.: Quantum Mechanics. North-Holland, Amsterdam (1962)

41. Sarandy, M.S., Lidar, D.A.: Adiabatic approximation in open quantum systems. Phys. Rev. A 71, 012331 (2005)

42. Ambainis, A., Regev, O.: An elementary proof of the quantum adiabatic theorem, pp. 1-12 (2004). arxiv:quant-ph/0411152v2

43. Sarandy, M.S., Wu, L.-A., Lidar, D.A.: Consistency of the adiabatic theorem. Quantum Inf. Process. 3, 331-349 (2004)

44. Amin, M.: Consistency of the adiabatic theorem. Phys. Rev. Lett. 102, 220401 (2009)

45. Tong, D.M.: Quantitative condition is necessary in guaranteeing the validity of the adiabatic approximation. Phys. Rev. Lett. 104, 120401 (2010)

46. Du, J., et al.: Experimental study of the validity of quantitative conditions in the quantum adiabatic theorem. Phys. Rev. Lett. 101, 060403 (2008)

47. Du, J., Li, H., Xu, X., Zhou, X., Han, R.: Phase-transition-like behaviour of quantum games. J. Phys. A Math. Gen. 36, 6551 (2003)

48. Hen, I.: Quantum gates with controlled adiabatic evolutions. Phys. Rev. A 91, 022309 (2015)

49. Meyer, D.A.: Quantum strategies. Phys. Rev. Lett. 82, 1052-1055 (1999)

50. Sun, C.-P.: High-order adiabatic approximation for non-Hermitian quantum system and complexification of Berry's phase. Phys. Scr. 48, 393 (1993)

51. Ibáñez, S., Muga, J.G.: Adiabaticity condition for non-Hermitian Hamiltonians. Phys. Rev. A 89, 033403 (2014)

52. Colin, E.C.: Pesquisa Operacional: 170 Aplicações em Estratégia, Finanças, Logística, Produção, Marketing e Vendas. LTC, Rio de Janeiro (2007)

53. Moreira, D.A.: Pesquisa Operacional: Curso Introdutório. Cengage Learning, São Paulo (2010)

54. Du, J., et al.: Experimental realization of quantum games on a quantum computer. Phys. Rev. Lett. 88, $137902(2002)$

55. Li, S.-B.: Simulation of continuous variable quantum games without entanglement. J. Phys. A Math. Theor. 44, 295302 (2011)

56. Johnson, M.W., et al.: Quantum annealing with manufactured spins. Nature 473, 194-198 (2011) 